\title{
Strategic Distractor Suppression Improves Selective Control in Human Vision
}

\author{
Wieske van Zoest, ${ }^{1,2}{ }^{-}$Christoph Huber-Huber, ${ }^{2,3}$ Matthew D. Weaver, ${ }^{2}$ and Clayton Hickey ${ }^{1,2}$ \\ ${ }^{1}$ School of Psychology and Centre for Human Brain Health, University of Birmingham, Birmingham B15 2TT, England, ${ }^{2}$ Centre for Mind/Brain \\ Sciences, University of Trento, 38068 Trento, Italy, and ${ }^{3}$ Donders Institute for Brain, Cognition, and Behaviour, Radboud University Nijmegen, \\ 6500 GL Nijmegen, The Netherlands
}

Our visual environment is complicated, and our cognitive capacity is limited. As a result, we must strategically ignore some stimuli to prioritize others. Common sense suggests that foreknowledge of distractor characteristics, like location or color, might help us ignore these objects. But empirical studies have provided mixed evidence, often showing that knowing about a distractor before it appears counterintuitively leads to its attentional selection. What has looked like strategic distractor suppression in the past is now commonly explained as a product of prior experience and implicit statistical learning, and the long-standing notion the distractor suppression is reflected in $\alpha$ band oscillatory brain activity has been challenged by results appearing to link $\alpha$ to target resolution. Can we strategically, proactively suppress distractors? And, if so, does this involve $\alpha$ ? Here, we use the concurrent recording of human EEG and eye movements in optimized experimental designs to identify behavior and brain activity associated with proactive distractor suppression. Results from three experiments show that knowing about distractors before they appear causes a reduction in electrophysiological indices of covert attentional selection of these objects and a reduction in the overt deployment of the eyes to the location of the objects. This control is established before the distractor appears and is predicted by the power of cue-elicited $\alpha$ activity over the visual cortex. Foreknowledge of distractor characteristics therefore leads to improved selective control, and $\alpha$ oscillations in visual cortex reflect the implementation of this strategic, proactive mechanism.

Key words: alpha; attention; distractor positivity; distractor suppression; electroencephalogram; N2pc

Significance Statement

To behave adaptively and achieve goals we often need to ignore visual distraction. Is it easier to ignore distracting objects when we know more about them? We recorded eye movements and electrical brain activity to determine whether foreknowledge of distractor characteristics can be used to limit processing of these objects. Results show that knowing the location or color of a distractor stops us from attentionally selecting it. A neural signature of this inhibition emerges in oscillatory alpha band brain activity, and when this signal is strong, selective processing of the distractor decreases. Knowing about the characteristics of task-irrelevant distractors therefore increases our ability to focus on task-relevant information, in this way gating information processing in the brain.

\section{Introduction}

This article addresses two linked and contentious issues in our understanding of visual attention. The first concerns our ability to strategically and proactively suppress distractors. Can we

\footnotetext{
Received Mar. 16, 2021; revised May 28, 2021; accepted June 29, 2021.

Author contributions: W.v.Z., C.H.-H., M.D.W., and C.H. designed research; C.H.-H. and M.D.W. performed research; C.H. analyzed data; W.v.Z. and C.H. wrote the paper.

This work was supported by an H2020 European Research Council Starting Grant (804360-INSENSE) to C.H. We thank Oscar Ferrante, Ole Jensen, and Damiano Grignolio for discussion and Hanna Kadel for discussion and research support.

The authors declare no competing financial interests.

Correspondence should be addressed to Wieske van Zoest at w.vanzoest@bham.ac.uk or Clayton Hickey at c.m.hickey@bham.ac.uk.

https://doi.org/10.1523/JNEUROSCI.0553-21.2021

Copyright $\odot 2021$ the authors
}

volitionally limit processing of stimuli that we know will be task irrelevant before they appear? The second issue regards the relationship between distractor suppression and alpha band oscillatory brain activity. If proactive suppression is possible, is it reflected in $\alpha$ ?

Extant evidence for strategic distractor suppression is mixed. Studies have shown that cues identifying distractor locations speed target responses (Awh et al., 2003; Munneke et al., 2008; Chao, 2010), cause eye movements to deviate from the inhibited location (van der Stigchel and Theeuwes, 2006), reduce the need for inhibitory processing when the distractor appears (Heuer and Schubö, 2019), and elicit anticipatory activity in the retinotopic visual cortex (Ruff and Driver, 2006; Munneke et al., 2011). But other work shows that distractor cues lead to monitoring of the cued location (Wang and Theeuwes, 2018), so that 
A Experiment 1

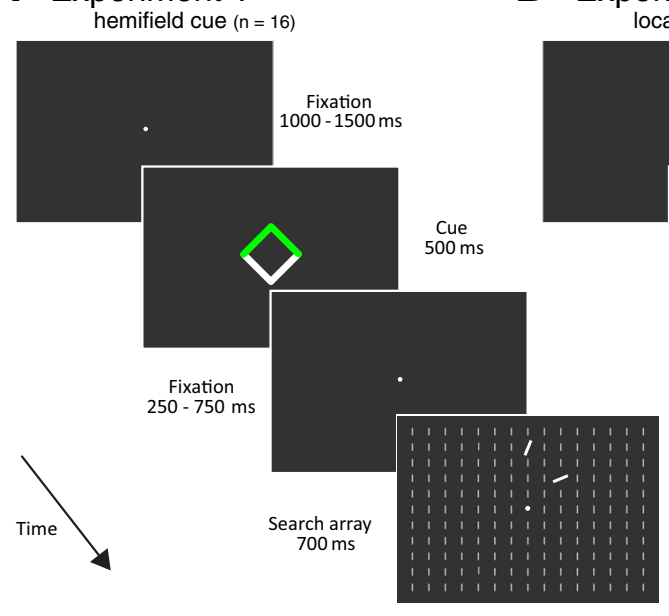

B Experiment 2

ocation cue $(n=14)$

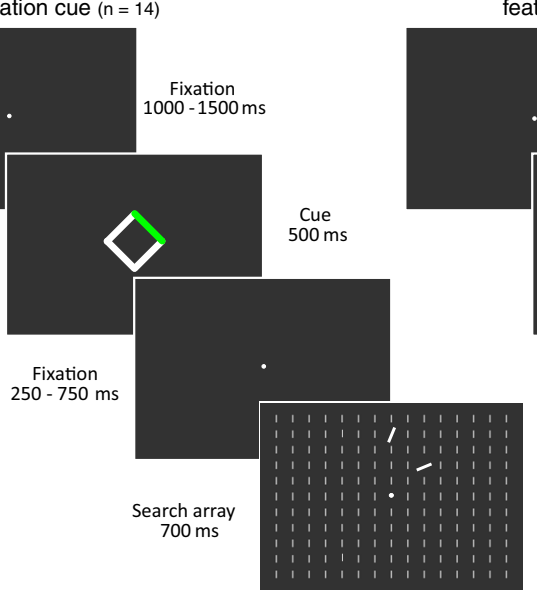

C Experiment 3

feature cue $(n=14)$
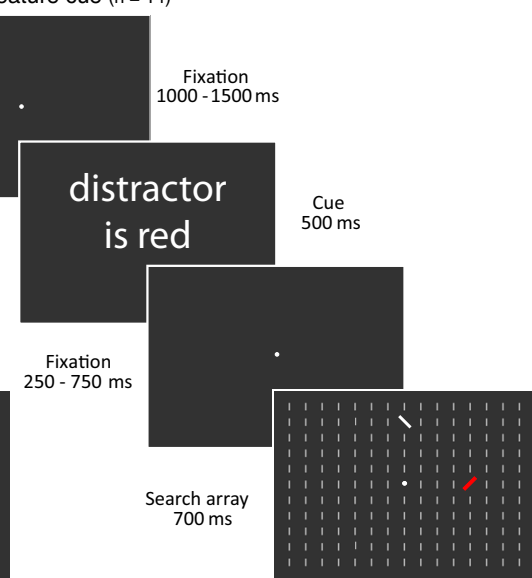

Figure 1. Schematics of cue trials. $\boldsymbol{A}$, In experiment 1 , the green elements of the cue indicated that the distractor would appear at one of two lateral locations in the upper visual hemifield or at one of two lateral locations in the lower visual hemifield. $\boldsymbol{B}$, In experiment 2 , the green element identifies the specific location where the distractor would appear. $\boldsymbol{C}$, In experiment 3 , the cue indicated that a red distractor would appear. Within each experiment, these cue conditions were contrasted with conditions in which the cue was uninformative. Note that the size of the cues are not to scale, that the semantic cue in experiment 3 was actually a single word (distractor or ready), and that the salient target and distractor were of the same size as background line elements in the actual experiment.

information from stimuli at this location intrudes into visual perception (Chang et al., 2019), and that neural signatures of distractor inhibition fail to emerge when there is no target to select (Hilimire et al., 2012).

Studies of feature cuing are no less confusing. Foreknowledge of distractor features, like color, can elicit preparatory activity in the visual cortex (Reeder et al., 2017). This appears to benefit the search for a target (Woodman and Luck, 2007; Arita et al., 2012) by facilitating suppression of the distractor after it appears (Carlisle and Woodman, 2011; de Vries et al., 2019). But this is not consistently observed (Becker et al., 2015), and other work shows that maintaining mnemonic representations of distractors causes these stimuli to draw attention (Moher and Egeth, 2012; Cunningham and Egeth, 2016; Beck et al., 2018). Part of the confusion stems from the fact that distractor suppression can be created by implicit learning (Cunningham and Egeth, 2016; Noonan et al., 2016; Ferrante et al., 2018; Wang and Theeuwes, 2018; van Moorselaar and Slagter, 2019), and this is often confounded with strategy in experimental designs. Recent reviews unanimously conclude that proactive distractor inhibition emerges as a product of prior experience, expectations, and implicit learning but that evidence for strategic distractor suppression is unconvincing (Noonan et al., 2018; Chelazzi et al., 2019; Gaspelin and Luck, 2019; van Moorselaar and Slagter, 2020; Luck et al., 2021).

A deep body of literature has linked distractor suppression with the emergence of alpha band oscillatory brain activity, but as broad doubt has grown regarding proactive distractor suppression generally, questions regarding the relationship between $\alpha$ and suppression have also emerged. Alpha $(\sim 8-12 \mathrm{~Hz})$ is clearly linked to neural inhibition; it predicts decreases in neural spiking (Haegens et al., 2011), $\gamma$ band activity (Spaak et al., 2012), and functional magnetic resonance imaging (fMRI) signals (Scheeringa et al., 2012). Evocatively, a cue identifying the location of a forthcoming target causes $\alpha$ to increase in the ipsilateral hemisphere, which represents the physical area where no relevant stimulus will appear (Worden et al., 2000; Thut et al., 2006). As $\alpha$ laterality increases, the representation of stimuli in the unattended field degrades (Händel et al., 2011). This sort of finding has led to the influential proposal that $\alpha$ reflects a neural process-perhaps the phasic activation of inhibitory GABAergic interneurons - that stops the propagation of unattended visual information in the retinotopic cortex (Klimesch et al., 2007; Jensen and Mazaheri, 2010; Foxe and Snyder, 2011). But neural inhibition as a basic mechanism may instantiate computational processes linked to target resolution rather than distractor suppression, and recent work has failed to find a relationship between $\alpha$ and behavioral indices of distractor suppression created through implicit learning (Noonan et al., 2016; van Moorselaar and Slagter, 2019). Prominent theoretical reviews suggest that the relationship between $\alpha$ and distractor suppression is unconvincing and that $\alpha$ is likely to reflect target processing (Noonan et al., 2018; Foster and Awh, 2019; van Moorselaar and Slagter, 2020).

Here, we use the concurrent recording of EEG and eye movements to test the notion of strategic, proactive distractor suppression and to identify $\alpha$ 's role in this cognitive mechanism.

\section{Materials and Methods}

Experiment 1 was designed to measure the impact of a spatial distractor cue on ERP evidence of selective processing, as reflected in the posterior lateral components N2pc (Luck and Hillyard, 1994a) and distractor positivity ( $\mathrm{P}_{\mathrm{D}}$; Hickey et al., 2009). To foreshadow, experiment 1 demonstrates that a spatial cue elicits $\alpha$ over the posterior lateral cortex and reduces selective processing of the distractor. Experiment 2 builds from this finding to test whether variance in distractor selection is predicted by the power of cue-elicited lateral $\alpha$. Experiment 3 extends both earlier studies to investigate the impact of a semantic cue identifying the distractor color.

In experiments 1 and 2, the target always appeared directly above or below fixation, and the distractor always appeared at one of four lateral locations (to the left or right in the upper or lower hemifield; Fig. 1). There are critical implications of this layout. First, it meant that the distractor cue in no way limited the scope of possible target locations, removing the possibility that the distractor cue provided information about the location of the target.

Second, this layout meant that targets were always on the vertical meridian, and distractors were always lateral. Locations on the vertical meridian of the visual field are equally represented in the two visual cortices, so processing of these locations generates equal activity across the brain hemispheres. In contrast, lateral locations are initially represented in the contralateral hemisphere, so visual processing of these 
locations generates lateralized brain activity. By presenting targets at vertical positions and distractors at lateral positions, we were able to identify lateralized brain activity linked to distractor processing when it was not confounded with activity linked to target processing (Woodman and Luck, 2003; Hickey et al., 2006, 2009).

Third, this layout meant that the target and distractor appear near to one another in the same upper or lower visual hemifield, or distant from one another in separate upper and lower hemifields. Distractor interference is known to scale as a function of the proximity of target and distractor stimuli (Mounts, 2000; Hickey and Theeuwes, 2011; Mathôt et al. 2010), and our expectation was that participants might be particularly motivated to employ proactive suppression when the experimental design contained conditions where target and distractor could activate a similar pool of retinotopic neurons. This circumstance is thought to create ambiguity in representation, competition for neural resources, and increased need for attentional mechanisms (Desimone and Duncan, 1995; Luck et al., 1997b).

Finally, this layout created a consistent relationship between location and distractor status. Recent results suggest that distractor suppression may develop through prior experience (Ferrante et al., 2018; Wang and Theeuwes, 2018; van Moorselaar and Slagter, 2020). Our stimulus layout provided preconditions potentially required for the initial development of distractor suppression, which may be necessary for subsequent strategic recruitment of this mechanism. Similar logic applied in experiment 3 , where a red color more often characterized the distractor than it did the target. This gave an extended opportunity for participants to become familiar with the need to suppress red stimuli, allowing us to answer the question of whether, once established, distractor suppression could be proactively recruited.

\section{Participants}

Seventeen volunteers ( 4 male, 23.2 years $\pm 2.7 \mathrm{SD}, 2$ left-handed) completed experiment 1,15 volunteers ( 3 male, 22.1 years \pm 2.2 SD, 2 lefthanded) completed experiment 2 , and 14 volunteers completed experiment 3 ( 4 male; 23.2 years, SD $=2.7$ years, 2 left-handed). One participant from each of experiments 1 and 2 was excluded from consideration because of low task accuracy resulting in too few trials for analysis $(>2$ $\mathrm{SD}$ from group mean). All participants had normal or corrected-to-normal vision, none reported any neurologic or psychiatric disorder, none took part in more than one of the experiments, and each was paid 10 Euros per hour of participation. The study was approved by the local ethics committee and conducted in accordance with the Declaration of Helsinki.

\section{Stimuli and procedure}

As illustrated in Figure 1, the stimuli and procedure for each of the experiments were similar. Each trial began with a requirement for participants to fixate a central mark, which was verified via eye tracking. Participants then initiated the stimuli sequence by pressing the space bar of a standard keyboard, which also triggered a drift-correction procedure in the eye-tracking software. The central fixation subsequently appeared alone for a random duration of 1000-1500 ms (uniform distribution), followed by appearance of the cue for $500 \mathrm{~ms}$, return of the fixation cross for 250-750 ms (uniform distribution), and appearance of the search array for $700 \mathrm{~ms}$. The search array consisted of a $15 \times 15$ square array of white line elements surrounding the central fixation mark (van Zoest et al., 2004; Fig. 1). The array subtended $\sim 27^{\circ} \times 27^{\circ}$ visual angle, and each element was $0.1^{\circ} \times 1^{\circ}$. Two elements in the array-the target and distractor-had an off-vertical orientation. The participant's task was to make a saccadic eye movement to the target line element.

In experiments 1 and 2, the target was defined both by degree of rotation and location. The target was rotated $22.5^{\circ}$ to the right and appeared $7.7^{\circ}$ (visual angle) directly above or below fixation, whereas the distractor was oriented $67.5^{\circ}$ to the right and was located $5.4^{\circ}$ (visual angle) from the fixation mark along a path tilted $45^{\circ}$ from the vertical path between fixation and target (Fig. $1 A, B)$. A diamond-shaped cue $\left(1.5^{\circ} \times 1.5^{\circ}\right.$ visual angle) identified where the distractor would appear in $50 \%$ of trials. In experiment 1, the upper or lower half of the otherwise white [red, green, blue (RGB): 200, 200, 200) cue could have a green color, indicating that the distractor would appear in one of two bilateral locations in the upper or lower visual hemifield. In the cue trials of experiment 2, only one line segment of the diamond was green, and this identified the precise distractor location. In remaining trials the distractor remained entirely white, providing no information about the distractor location. This is referred to as the no-cue condition below.

In experiment 3 the target was defined by direction of rotation. Both the target and distractor line segments were $45^{\circ}$ off vertical, rotated in opposite directions, and half of the participants were instructed to make a saccade to the left tilted line element and the rest to the right tilted element. The target and distractor could appear at one of four equidistant locations $7.7^{\circ}$ (visual angle) to the left/right of fixation or above/below fixation. When the target appeared at locations on the vertical meridian of the display, the distractor appeared to the left or right of fixation, whereas when the target appeared to the left or right of fixation, the distractor necessarily appeared on the vertical meridian. In $66 \%$ of trials, the distractor had a unique red color (RGB: 250, 0, 0; Fig. 1C). In the remaining trials the target had the unique red color. The semantic cue was either the word "ready" ("pronto" for Italian-language participants) or "distractor." The ready cue appeared in two-thirds of trials and indicated that either the target or the distractor could have the unique red color with equal probability. The distractor cue appeared in one-third of trials and indicated that the distractor would be uniquely identified by the red color. The cue appeared at fixation in a 14-point font.

Experiments 1 and 2 consisted of 1 practice block of 64 trials followed by 12 experimental blocks of 64 trials. This took $\sim 2 \mathrm{~h}$ to complete, including $1 \mathrm{~h}$ of EEG preparation. Experiment 3 was longer, consisting of 1 practice block of 32 trials followed by 18 blocks of 64 trials, and took $\sim 2.5 \mathrm{~h}$, including $1 \mathrm{~h}$ of EEG preparation. For all experiments, stimuli were presented on a $57 \mathrm{~cm}$ VIEWPixx LCD monitor $(120 \mathrm{~Hz})$ with a viewing distance of $1 \mathrm{~m}$. The experiments were prepared using Psychtoolbox-3 (Brainard, 1997).

\section{Eye tracking and EEG recording}

Eye movement and EEG data were simultaneously recorded in all experiments. A desk-mounted Eyelink 1000 (SR Research) recorded monocular eye position at $1 \mathrm{kHz}$. In most cases tracking was of the right eye, but occasionally the left eye was used when this generated better eye-tracker calibration.

EEG was recorded at $1 \mathrm{kHz}$ from 62 cap-mounted $\mathrm{Ag} / \mathrm{AgCl}$ electrodes in a 10/20 montage and 2 electrodes mounted over the left and right mastoids. Electrode impedances were kept below $10 \mathrm{k} \Omega$ during recording. EEG was amplified with a BrainAmp amplifier (BrainProducts) with right-mastoid reference and subsequently rereferenced to the average of both mastoid signals. Analog anti-alias filters were applied during recording $(0.016-250 \mathrm{~Hz})$, and the data were subsequently digitally lowpass filtered using a 101-point zero-phase noncausal least-square finite impulse response kernel $(0 \mathrm{~dB}$ attenuation at $40 \mathrm{~Hz} ;-6 \mathrm{~dB}$ at $45 \mathrm{~Hz})$.

\section{Data analysis}

Analysis was conducted with custom scripts for MATLAB R2020a (MathWorks), which used the EEGLAB toolbox (Delorme and Makeig, 2004), the EYE-EEG toolbox (Dimigen et al., 2011), the FieldTrip toolbox (Oostenveld et al., 2011), and the MATLAB Statistics toolbox (version 11.7). Statistical analysis relied on parametric ANOVAs, permutation contrasts, and mixed linear modeling. In permutation contrasts, the null distribution is based on $10^{5}$ random samples of the effect of interest with replacement. In mixed linear modeling, maximum likelihood is used for variance estimation, Akaike information criterion (AIC) is used for model comparison, and ANOVA derivations employ Satterthwaite approximations of degrees of freedom.

Eye Movements. Eye movements with a velocity exceeding $30 \%$ or acceleration exceeding $8000 \% \mathrm{~s}^{2}$ were marked as saccades. Saccadic response times (SRTs) were calculated as the time between stimulus onset and the beginning of the first saccade larger than $3^{\circ}$ (visual angle). The saccade was recorded as target directed or distractor directed when it landed within $2.7^{\circ}$ (visual angle) of the center of these stimuli.

In experiment 1 , saccade trajectory deviations were quantified as the mean angular deviation from a straight-line path between the saccade 

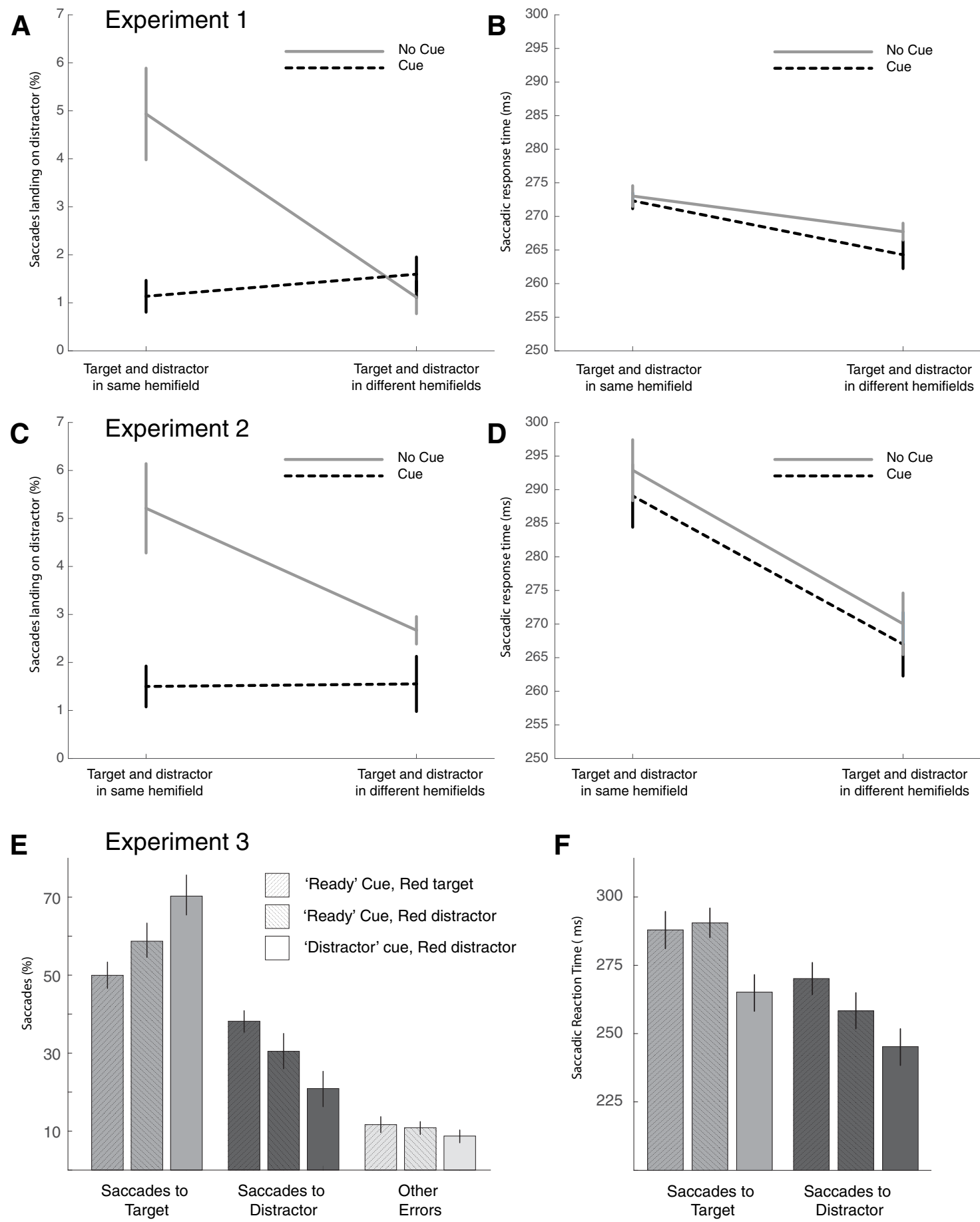

Figure 2. Behavioral results. $A$, Saccadic performance in experiment 1. When the target and distractor appeared in the same visual hemifield, the spatial cue reduced the proportion of saccades landing on the distractor. B, Saccadic response times in experiment 1. C, Saccadic performance in experiment 2. Results from experiment 1 are broadly reproduced. D, Saccadic response times in experiment 2. $\boldsymbol{E}$, Saccadic performance in experiment 3. The distractor cue reduced the proportion of saccades landing on the distractor. $\boldsymbol{F}$, Saccadic response times in experiment 3. The distractor cue reduced saccadic response times in accurate trials. All error bars reflect within-participant SEM (Cousineau, 2005).

starting point and the center of the targets for each eye-tracker sample (Van der Stigchel et al., 2006). The first five samples of the saccade were excluded from this calculation. Negative saccade deviation values reflect deviation away from the distractor location. To illustrate mean saccades, linear interpolation was used to generate representations of each saccade with an equal number of samples. Within each condition these interpolated saccades were mean averaged across trials, and subsequently across participants, to generate the saccade paths (see Fig. 3).
EEG. Infomax independent component analysis (ICA; Bell and Sejnowski, 1995) was conducted on combined EEG and eye position data. Artefactual independent components were identified based on their covariance with eye movement data using a saccade-to-fixation variance criterion of 1.1 (Plöchl et al., 2012), and variance associated with these components was removed from the EEG. Epochs were created in an interval beginning $1 \mathrm{~s}$ before stimulus onset and ending $1 \mathrm{~s}$ after. ERPs were calculated from mean signal in a cluster of lateral posterior electrode sites where the N2pc (Luck and Hillyard, 1994a,b) and $\mathrm{P}_{\mathrm{D}}$ (Hickey 

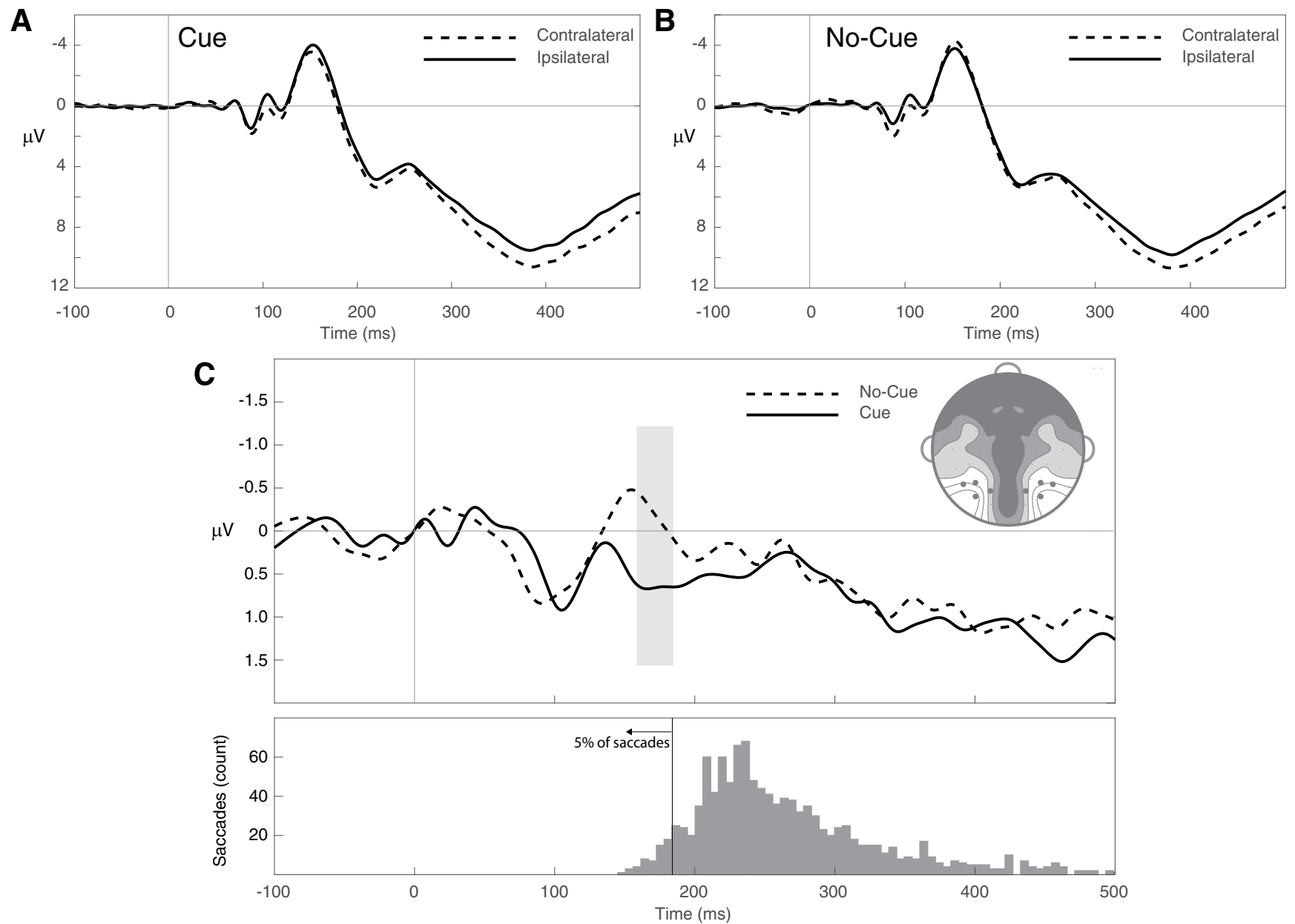

Figure 3. ERPs from experiment 1, time locked to onset of the search array, when the array contained a target and distractor in the same upper or lower visual hemifield. $A$, Posterior lateral ERPs elicited contralateral and ipsilateral to the location of a cued distractor. $\boldsymbol{B}$, Posterior lateral ERPs elicited contralateral and ipsilateral to the location of an uncued distractor. $\boldsymbol{C}$, Contralateral-minus-ipsilateral difference waves. Illustrated in the bottom panel is the distribution of saccadic response times. The cue caused a positive shift in the difference wave immediately before the onset of saccadic responses. Topography illustrates mean voltage difference from the interval identified by shading. Because the effect is a difference in contralateral-minus-ipsilateral waveforms, the lateral topography is represented in both hemispheres with midline electrodes set to zero value.

et al., 2009) are maximal (PO3/4, PO7/8, P5/6, and P7/8) and baseline corrected on an interval beginning $100 \mathrm{~ms}$ before stimulus onset and ending $50 \mathrm{~ms}$ after. Only correct trials were included in ERP analysis.

Oscillatory analysis of prestimulus EEG was used to index the effect of the spatial cue before the onset of target and distractor stimuli. This relied on the application of Gabor transforms to data observed in correct trials. Kernel cycles increased linearly from one cycle at $4 \mathrm{~Hz}$ to eight cycles at $40 \mathrm{~Hz}$, so that kernels had a length of $221 \mathrm{~ms}$ at $8.3 \mathrm{~Hz}, 215 \mathrm{~ms}$ at $10 \mathrm{~Hz}$, and $213 \mathrm{~ms}$ at $12.5 \mathrm{~Hz}$. The conditional difference in oscillatory power was computed between cuing conditions (with no other baseline) before being mean averaged across a cluster of electrodes over the lateral posterior cortex (see Fig. 6). The electrode clusters corresponded to those used for ERP analysis and are consistent with those used in earlier studies of lateral $\alpha$ and attention (Worden et al., 2000). In experiment 2, lateral power was subsequently computed by subtracting ipsilateral values from contralateral values. In experiments 1 and 3, bilateral power was calculated by averaging across the sets of lateral electrodes. Cluster-based permutation tests were used to test the effect of the cue on oscillatory power. Clusters were defined across frequency and latency dimensions with a cluster-defining threshold of $p<0.05$ and cluster significance at $p<$ 0.05 . In experiments 2 and 3 , generation of the null distribution relied on exhaustive relabeling of cue and no-cue conditions $\left(2^{14}\right.$ iterations), and in experiment 1 relied on a random sample of $2^{14}$ relabelings of cue and no-cue conditions (of the $2^{16}$ combinations possible). Statistical analysis was conducted across a time range beginning $850 \mathrm{~ms}$ before stimulus onset and ending $850 \mathrm{~ms}$ after and across a frequency range beginning at $4 \mathrm{~Hz}$ and ending at $40 \mathrm{~Hz}$.

\section{Results}

\section{Experiment 1}

In experiment 1 , the distractor cue identified two bilateral locations in the upper or lower visual hemifield where the distractor could appear (Fig. 1A). This hemispheric cue was used to ensure that the cue did not elicit lateralized activity that could sustain into the post-target interval and complicate interpretation of activity evoked by the distractor itself.

\section{Behavior}

Trials were removed from analysis if the eyes were not at fixation at the trial start $(3.8 \%$ of trials), if the saccade was anticipative (SRT of $<60 \mathrm{~ms} ; 0.3 \%$ ), or if the eyes landed elsewhere than on the target or distractor $\left(>2.7^{\circ}\right.$ visual angle from center of object; $1.5 \%)$.

As illustrated in Figure $2 A$, when the target and distractor appeared together in the upper or lower visual hemifield, the cue reduced the proportion of saccades that were deployed to the distractor. This effect did not emerge when the stimuli were presented in opposite visual hemifields. To test this, we conducted a two-way repeated-measures ANOVA (RANOVA) with factors 

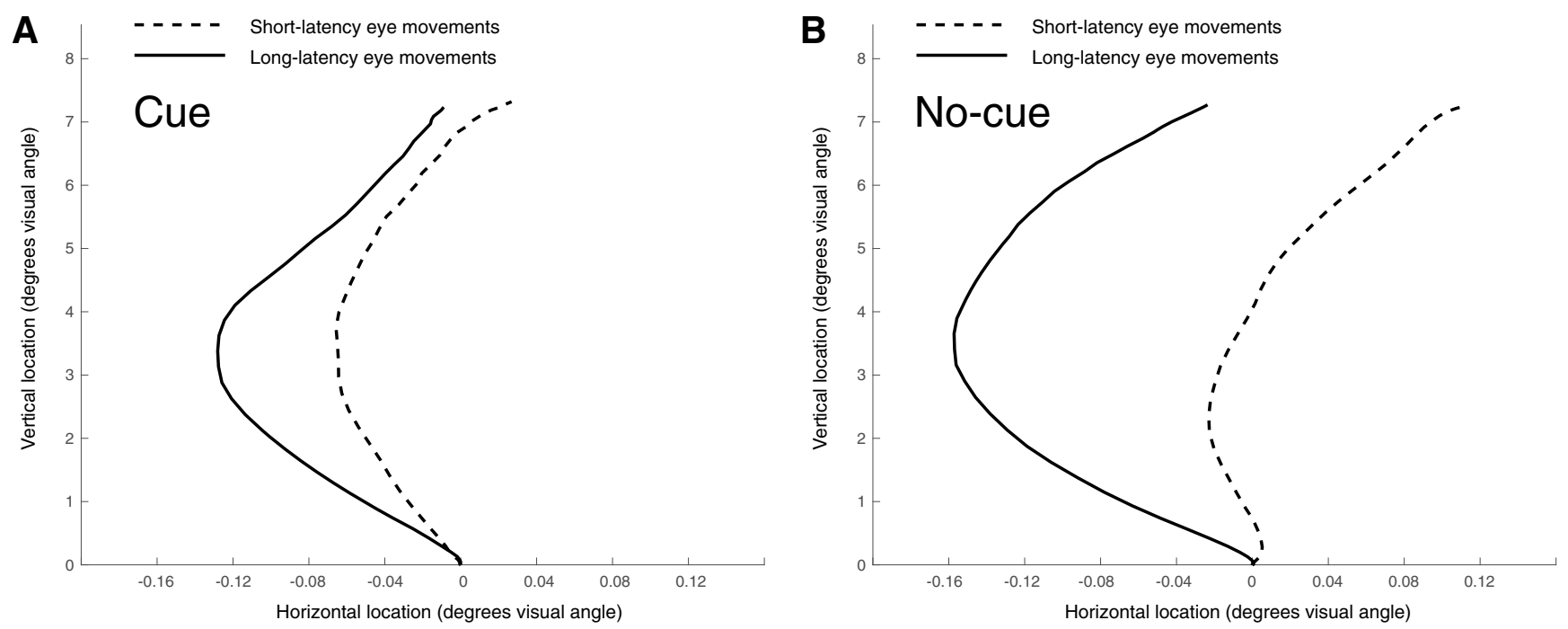

Figure 4. Saccadic deviation results from experiment 1. Note that although the target could appear above or below fixation, and the distractors to the left or right and above or below fixation, these images have been rectified so that the illustrative target location is above fixation (at origin) and the distractor is to the right. $A$, Mean short-latency and long-latency saccades following a distractor cue. $\boldsymbol{B}$, Mean short-latency and long-latency saccades following a neutral cue. In the neutral cue condition short-latency saccades deviate strongly toward the distractor, whereas long-latency saccades deviate strongly away from the distractor, describing a time course of distractor inhibition. This time course is reduced in the cue condition.

for shared hemisphere (same hemisphere vs different hemispheres) and cue (cue vs no-cue). This identified the main effects of cue $\left(F_{(1,15)}=6.32, p=0.023\right)$ and shared hemisphere $\left(F_{(1,15)}=\right.$ $6.29, p=0.023)$ and an interaction between the factors $\left(F_{(1,15)}=\right.$ $13.76, p=0.002)$.

Figure $2 B$ illustrates SRTs as a function of the same factors. Analysis identified a main effect of shared hemisphere $\left(F_{(1,15)}=\right.$ 10.24, $p=0.006)$, reflecting slower SRTs when the stimuli appeared in the same visual hemisphere. No other significant effects emerged (cue: $F_{(1,15)}=1.55, p=0.232$; interaction $F<1$ ).

\section{ERPs}

Our interest lay in lateral effects over the occipital cortex in the time range of the N2pc (Luck and Hillyard, 1994a,b) and $\mathrm{P}_{\mathrm{D}}$ (Hickey et al., 2009). These lateral ERP components track attentional selection and stimulus-triggered distractor suppression, respectively. Figure 3 presents the ERPs elicited over the lateral occipital cortex ipsilateral and contralateral to the distractor when the distractor appeared in the same visual hemifield as the target. Results show that the cue affects the laterality of the visual ERP in the range of the $\mathrm{N} 2 \mathrm{pc}$ and $\mathrm{P}_{\mathrm{D}}$. When the distractor hemifield was cued, the contralateral waveform becomes more positive than the ipsilateral waveform from $\sim 150 \mathrm{~ms}$ post-target (Fig. $3 A$ ). In the no-cue condition, the contralateral waveform in the same interval is more negative than the ipsilateral waveform (Fig. 3B). These lateral effects are small in magnitude compared with the bilateral morphology of the ERP and are therefore more clearly illustrated in the contralateral-minus-ipsilateral difference waves presented in Figure $3 C$. From $\sim 150$ to $\sim 250 \mathrm{~ms}$ post-target, the difference wave elicited in the no-cue condition is more negative than is the difference wave elicited in the cue condition, reflecting stronger attentional selection of the distractor in the no-cue condition.

We focused statistical analysis on the interval immediately preceding the onset of saccadic responses. Following our earlier work, we operationally defined this as the $25 \mathrm{~ms}$ preceding the fifth percentile of the distribution of eye movements (165190 ms; Fig. 3C; Weaver et al., 2017). This ensured that the electrophysiological signal was not tainted by physiological artefacts of the eye movement that may have survived ICA correction and, more importantly, that the brain activity reflected in the signal preceded overt selective behavior and thus had the opportunity to play a role in determining that behavior.

Mean ERP voltage in this interval was assessed in a four-way RANOVA with factors for electrode laterality (ipsilateral versus contralateral), cue (cue vs no-cue), and shared visual hemifield (same hemifield versus opposite hemifield). An additional factor representing a median split of data based on saccadic response speed was included to equate these ERP analyses with analysis of saccade deviation described below (fast vs slow). The critical result was an interaction of electrode laterality, hemisphere, and cue $\left(F_{(1,15)}=5.093, p=0.039\right.$; Fig. $\left.3 C\right)$, reflecting relative positivity of the contralateral-minus-ipsilateral difference wave in cued trials, relative to no-cue trials, when the target and distractor were presented in the same visual hemifield rather than different hemifields. To follow up on this interaction, we contrasted the mean voltage of the difference waves for cue and no-cue conditions when the target and distractor were in the same or opposite visual hemifields using separate permutation tests. When target and distractor were presented in the same hemifield, this difference was significant ( $p=0.004$; Fig. $3 C$ ). When the target and distractor were presented in opposite hemifields, it was not ( $p=$ 0.583; not shown).

The RANOVA identified one other significant effect, an interaction of electrode laterality, shared hemisphere, and response speed $\left.\left(F_{(1,15}\right)=8.885, p=0.009\right)$. Importantly, this effect did not involve the critical manipulation of cue. This appears to reflect a propensity for the lateral distractor-elicited response to be more positive when target and distractor were presented in the same hemifield, and the response was slow rather than fast, but more negative when target and distractor were presented in opposite hemifields and the response was slow rather than fast. It may reflect a decrease in target-distractor interference and a propensity to broadly explore the environment when participants are relaxed about task completion. No other effect was significant (electrode laterality: $F_{(1,15)}=1.346, p=$ 0.264 ; saccadic response speed: $F_{(1,15)}=4.255, p=0.057$; cue: $F_{(1,15)}=1.129, p=0.305$; electrode laterality X cue: $F_{(1,15)}=1.709$, $p=0.211$; all other Fs $<1$ ). 


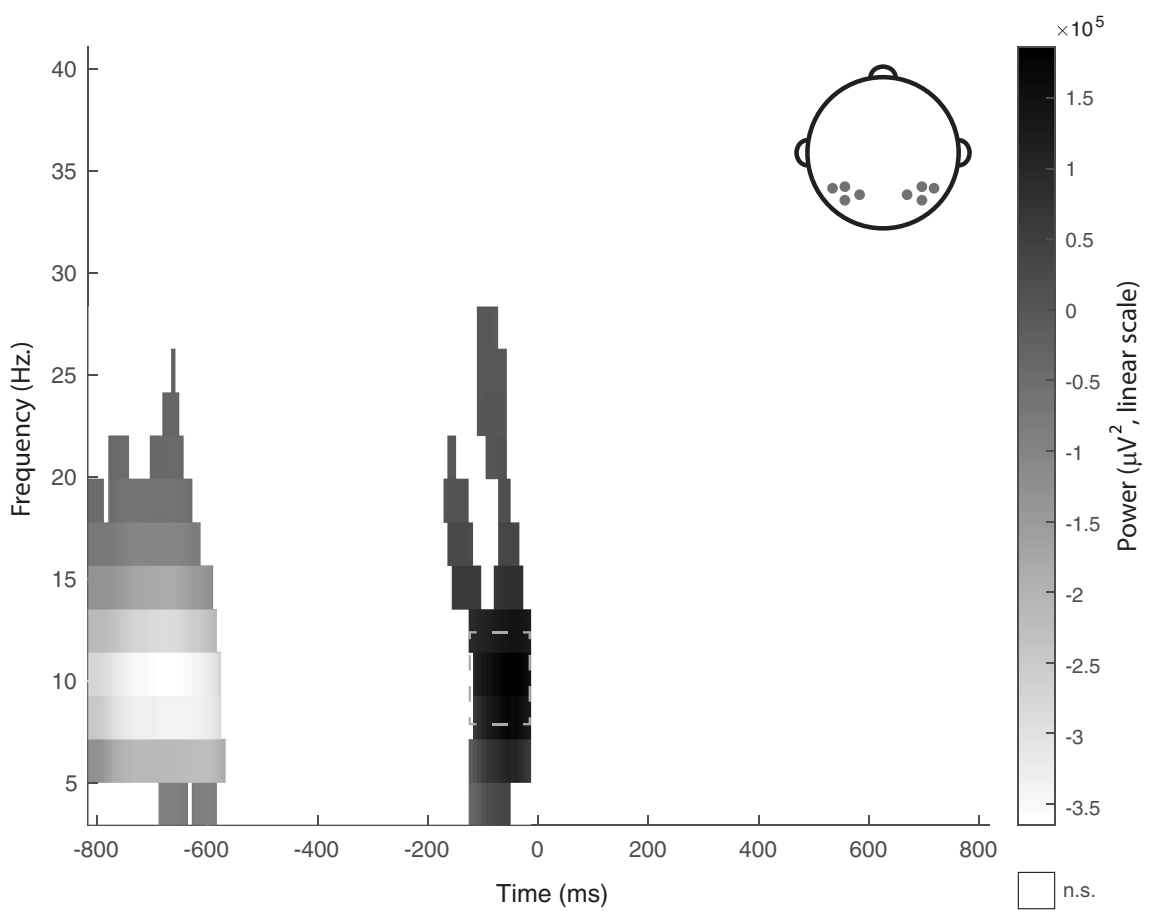

Figure 5. Posterior oscillatory power in Experiment 1 as observed in cue conditions with power observed in no-cue conditions subtracted. Shaded areas reflect time-frequency combinations where oscillatory power significantly differed between cue conditions. The time-frequency interval identified by broken box was used in trial-wise analysis of the relationship between oscillatory power and EEG amplitude. Results that did not survive statistical correction are identified with n.s.

The ERP and saccadic performance data from experiment 1 suggest one of two interpretations. The cued location may be proactively suppressed, causing stimuli appearing at that location to be less salient. As a result, the cued distractor does not draw attention and does not elicit an N2pc. This has an important implication: because distractor suppression would be fully implemented before the target and distractor stimuli appeared, it should be temporally stable in the post-target interval and not develop over time.

The alternative is that the cue primes a mechanism of stimulus-triggered distractor suppression - reflected in $\mathrm{P}_{\mathrm{D}}$ amplitude - that acts to negate distractor salience after the stimuli appear (Sawaki and Luck, 2010). By this interpretation, the cued distractor is actively suppressed in the post-target interval and elicits a $\mathrm{P}_{\mathrm{D}}$. The implication here is that distractor suppression develops within the post-target interval, and that distractor salience should accordingly be high soon after onset of the search array and reduce as the suppressive reaction builds.

\section{Saccade deviation}

We leveraged this distinction in time course expectations to clarify our interpretation of the ERP results from experiment 1 . The developmental time course of distractor suppression can be tracked in saccade deviation. Short-latency, target-directed eye movements show a tendency to deviate toward salient distractors during saccadic flight. But if the saccade is longer-latency-if more time has passed between stimulus onset and saccade onset -the saccade comes to deviate away from the distractor location (Mulckhuyse et al., 2009; Hickey and van Zoest, 2012; Weaver et al., 2017). This pattern has been linked to the development of a spatial inhibitory tag in the oculomotor system to resolve competition in neural representation (McPeek et al., 2003; Van der Stigchel et al., 2006). Saccade deviation can therefore be used to determine whether the proactive distractor suppression identified in experiment 1 is triggered by stimulus onset and therefore shows a time course in the post-target interval, or if it is implemented before stimulus onset and is therefore stable in the post-target interval.

Figure $4, A$ and $B$, presents results from analysis of saccade deviation in experiment 1 . Note that although the target could appear in the upper or lower hemifield, and the distractor to the left or right of fixation and above or below fixation, these plots have been rectified so the illustrative target location is in the upper visual hemifield, and the distractor appears to the right (Fig. 1A). In the no-cue condition, short-latency saccades (crossparticipant mean of per-participant conditional medians: $240 \mathrm{~ms}$ $\pm 31 \mathrm{~ms} \mathrm{SD}$ ) tended to deviate toward the distractor location, whereas long-latency saccades ( $307 \mathrm{~ms} \pm 46 \mathrm{~ms}$ SD) deviated away from the distractor location. The cue reduced this pattern: short-latency saccades ( $240 \mathrm{~ms} \pm 33 \mathrm{~ms}$ SD) showed less deviation toward the distractor than is observed in the no-cue condition, whereas long-latency saccades ( $311 \mathrm{~ms} \pm 53 \mathrm{~ms}$ SD) showed less deviation away.

To test this pattern, the mean deviation was entered into a RANOVA with factors for cue, saccadic response latency, and shared visual hemifield. This identified a critical interaction between cue and saccadic response latency $\left(F_{(1,15)}=6.116, p=\right.$ 0.026; hemisphere: $F_{(1,15)}=2.391, p=0.143$; speed: $F_{(1,15)}=$ 3.668, $p=0.075$; hemisphere $\times$ speed: $F_{(1,15)}=3.754, p=0.072$; all other $F$ s $<1$ ). Follow-up permutation contrasts identified that in no-cue trials, short-latency saccades differed in saccade deviation from long-latency saccades $(p=0.002)$, but in cue trials, there was no significant difference $(p=0.167)$. Results from saccade deviation thus show that (1) the cue reduced the initial salience of the distractor so that it did not draw eye movements initiated quickly, and (2) the cue removed the need for strong poststimulus suppression, so eye movements initiated after longer delays were not so strongly repelled away from the distractor. 

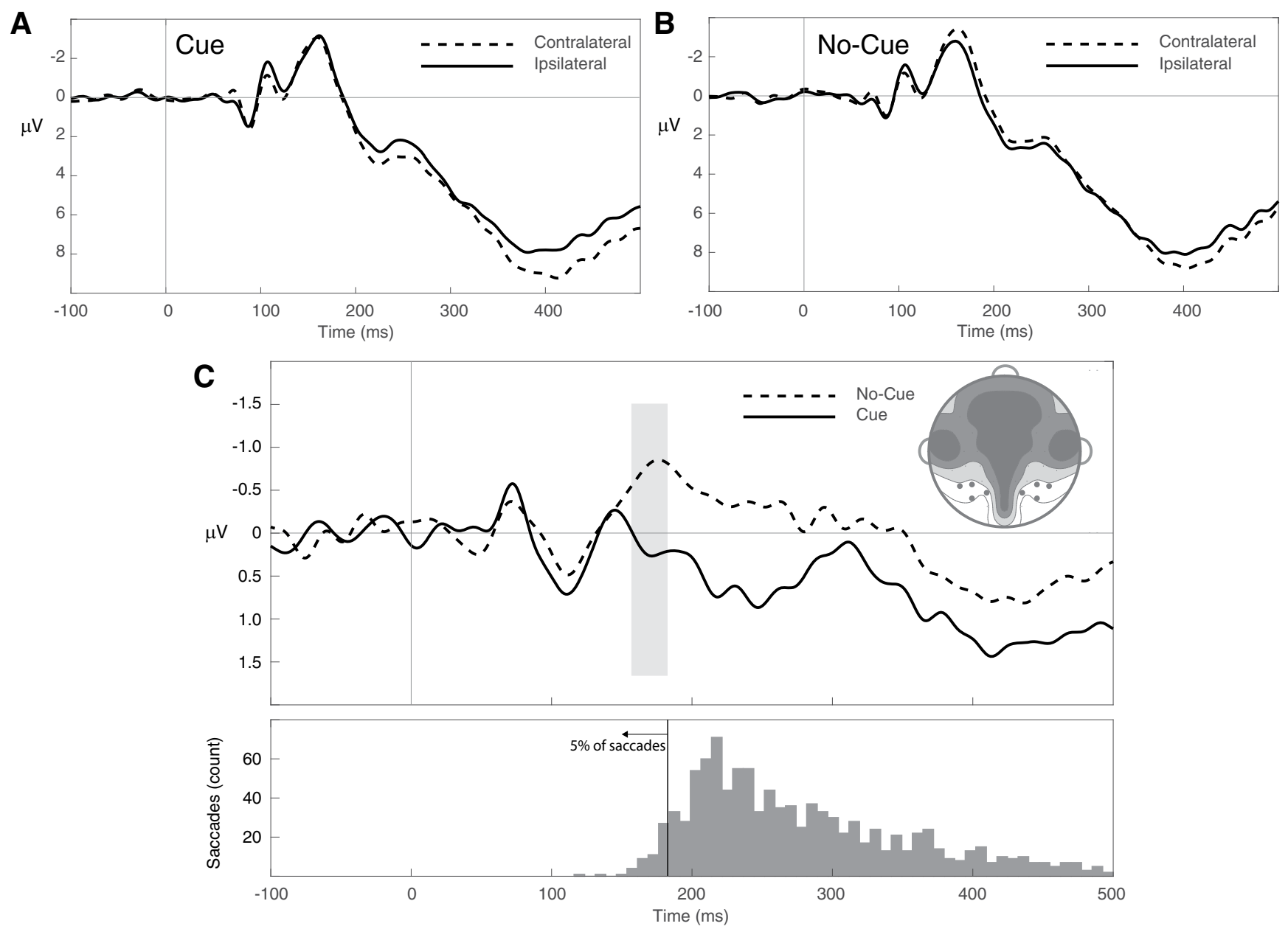

Figure 6. ERPs from experiment 2, time locked to the onset of the search array, when the array contained target and distractor in the same upper or lower visual hemifield. $\boldsymbol{A}$, Posterior lateral ERPs elicited contralateral and ipsilateral to the location of a cued distractor. $\boldsymbol{B}$, Posterior lateral ERPs elicited contralateral and ipsilateral to the location of an uncued distractor. $\boldsymbol{C}$, Contralateral-minus-ipsilateral difference waves. Illustrated in the bottom panel is the distribution of saccadic response times. Topography is calculated as described in Figure 3.

The cue attenuated the developmental time course of stimulustriggered distractor suppression, consistent with the idea that cue-elicited suppression was implemented before stimulus onset.

\section{Time-frequency analysis}

Time-frequency analysis focused on cue-elicited bilateral oscillatory power over the posterior occipital cortex. Results are illustrated in Figure 5. In the interval between the cue and search array, the cue created an initial decrease in oscillatory power in the alpha band, which was followed by an increase before onset of the search array. This is consistent with the idea that participants interpreted the informative cue, causing a decrease in $\alpha$, before subsequently implementing strategic suppression that is reflected in alpha power (Bonnefond and Jensen, 2012).

We approached results from experiment 1 with the idea that variance in cue-elicited bilateral $\alpha$ might predict selective processing in the post-target EEG, but we failed to find any evidence of this. Because we do find evidence of this relationship in experiments 2 and 3, details of this analysis are provided here. To test the relationship between $\alpha$ and EEG, we isolated cue trials and extracted trial-wise pretarget alpha power (over the timefrequency interval identified by the broken rectangle in Fig. 5) and trial-wise post-target lateral EEG amplitude (over the time interval identified by the gray rectangle in Fig. $3 C$ ). We rank transformed these values to reduce the impact of outlier values (Iman and Conover, 1979; Conover and Iman, 1981) and used mixed linear modeling and model selection to assess the relationship between cue-elicited $\alpha$ and distractor-elicited N2pc (using fitlme.m from the MATLAB statistics toolbox). A simple initial model included a continuous fixed effect predictor for alpha power (oscillatory_power) and a random effect for the intercept of each participant (participant; AIC: 47876) as follows:

$$
\mathrm{N} 2 \mathrm{pc} \sim \text { oscillatory_power }+(1 \mid \text { participant }) .
$$

We attempted to improve this model by adding all combinations of the following factors: random effects for the per-participant oscillatory power intercept and slope, a categorical fixed factor for the effect of distractor elevation in the visual field (top vs bottom), a categorical fixed factor for target location (top vs bottom), and a continuous fixed factor for SRT. The inclusion of distractor and target location was motivated by known variance in $\mathrm{N} 2 \mathrm{pc}$ and $\mathrm{P}_{\mathrm{D}}$ as a function of the elevation of the eliciting stimulus in the visual field (Hickey et al., 2009). None of these models performed better than the simple model described above. ANOVA analysis of this model failed to identify any relationship between bilateral $\alpha$ and distractor-elicited lateral EEG $\left(F_{(1,4125)}=\right.$ $0.302, p=0.583$ ). 

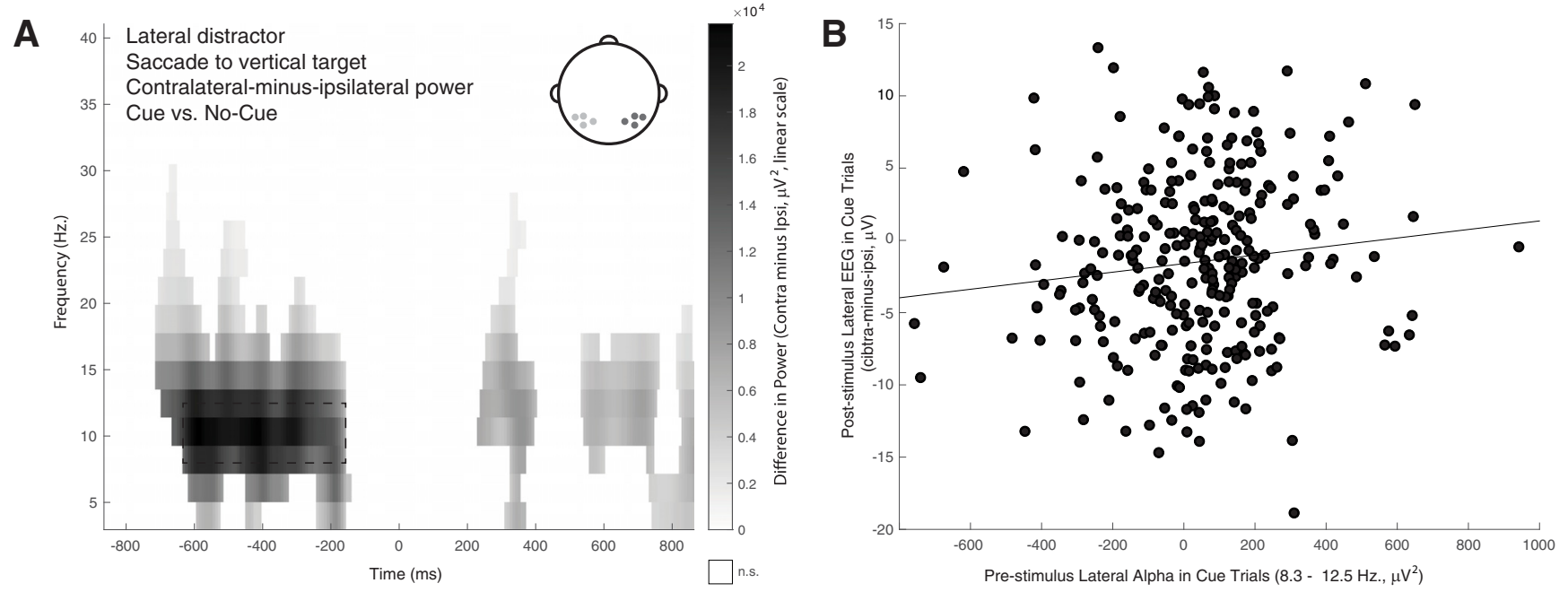

Figure 7. Time-frequency results from Experiment 2. $\boldsymbol{A}$, Lateral posterior oscillatory power. Shaded areas reflect time-frequency combinations where contralateral-minus-ipsilateral difference in oscillatory power was greater in the cue condition than the no-cue condition. The time-frequency interval identified by broken box was used in trial-wise analysis of the relationship between oscillatory power and EEG amplitude. Results that did not survive statistical correction are identified with n.s. B. Trial-wise relationship between pre-stimulus alpha and post-stimulus EEG amplitude for one illustrative participant. Note that while this illustration is of raw data, modelling was based on rank transformed values and included multiple predictive factors. Line reflects ordinary least-square linear fit.

Experiment 1 was designed to elicit strategic, proactive suppression of the cued distractor locations. However, there is the possibility that participants may have responded to the cue by deploying spatial attention to the target location in the countercued field. This strategy is unlikely as it would have no net benefit to task performance; it would make for easier programming of target-directed saccades in the $50 \%$ of trials where the target appeared at the monitored location but, equally, would make for more difficult target localization and saccade preparation in the $50 \%$ of trials where the target appeared on the other side of fixation. However, the results allow us to empirically assess the possibility. First, the deployment of spatial attention in response to a spatial cue is known to cause a broad and long-lasting decrease in bilateral $\alpha$ over occipital cortex (Sauseng et al., 2005; Thut et al., 2006; Dombrowe and Hilgetag, 2014). If participants deploy spatial attention to the countercued upper or lower visual hemifield, we should see a decrease of cue-elicited bilateral $\alpha$ in the cue condition compared with the nocue condition. In fact, our results show the opposite, with cue-elicited $\alpha$ increasing in this interval (Fig. 5). Second, if participants strategically deployed spatial attention to the countercued visual field, this should be motivated by a behavioral benefit when the target appears at the monitored location. Results from experiment 1 show no such spatial cuing effect on eye movements; when target and distractor appear in different hemifields, neither saccadic accuracy (Fig. 1A) nor SRT (Fig. 1B) differ between cue and no-cue conditions. Finally, although we have no clear expectations for how such a deployment of spatial attention would affect saccadic curvature, it is difficult to imagine how this could generate the pattern of saccade deviation we see in experiment 1 (Fig. 4). In contrast, the pattern of saccade deviation we observe is explicitly predicted by the notion of cue-elicited proactive inhibition.

\section{Experiment 2}

ERP results from experiment 1 identified variance in selective processing of the distractor as a function of the spatial distractor cue, and saccade deviation results suggested that cue-elicited suppression was implemented before onset of the search array. Time-frequency results identify a cue-elicited increase in bilateral alpha power, but this does not robustly predict subsequent distractor processing in the ERP. Bilateral $\alpha$ may be unsuited for this type of analysis as it can reflect neural mechanisms unrelated to spatial suppression of the cued location. Lateral $\alpha$, the difference of $\alpha$ across ipsilateral and contralateral hemispheres, provides better insight on spatial processing of the cued location, and with this in mind, experiment 2 used a cue that identified the discrete lateral location where a distractor would appear (Fig. 1B).

\section{Behavior}

Trials that did not begin with fixation (1.5\%), that were anticipative responses $(0.2 \%)$, or that did not result in target or distractor selection (7.6\%) were removed from analysis. As illustrated in Figure 2, $C$ and $D$, behavioral results replicated those observed in experiment 1; that is, when the target and distractor appeared together in the upper or lower visual hemifield, the cue reduced the proportion of saccades that were deployed to the distractor. This emerged in statistical analysis of saccadic performance as an interaction of cue and shared hemisphere $\left(F_{(1,13)}=14.13, p=\right.$ $0.002)$. The main effect of cuing also emerged $\left(F_{(1,13)}=8.13, p=\right.$ 0.014; shared hemisphere: $\left.F_{(1,13)}=2.54, p=0.135\right)$. Analysis of SRT identified main effects of shared hemisphere $\left(F_{(1,13)}=6.17\right.$, $p=0.027)$ and cue $\left(F_{(1,13)}=8.45, p=0.012\right.$; interaction $\left.F<1\right)$.

\section{ERPs}

As illustrated in Figure $6 A-C$, ERP results from experiment 2 also replicate experiment 1 . In the $25 \mathrm{~ms}$ interval preceding the fifth percentile of saccade onsets (158-183 ms), the cue caused a reduction in distractor-elicited N2pc. Building from experiment 1 , statistical analysis took the form of a single planned permutation contrast of the mean difference in ipsilateral-minus-contralateral voltage between cue and no-cue conditions in the 158$183 \mathrm{~ms}$ interval, limited to conditions where the target and distractor appeared in the same visual hemifield $(p=0.003)$. This effect did not emerge when the target and distractor appeared in different hemifields $(p=0.534)$. 


\section{Vertical red target / ‘Ready’ cue}

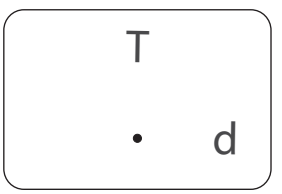

A Saccade to distractor

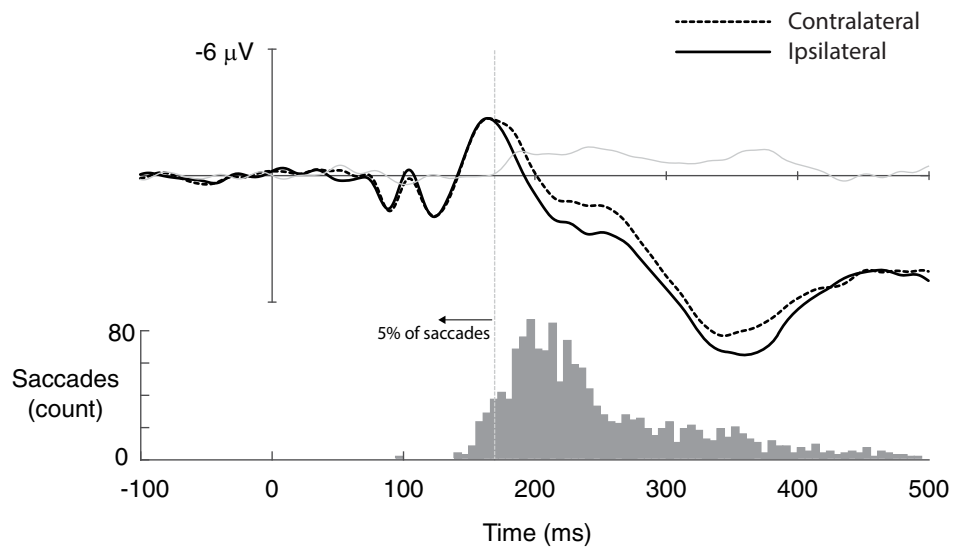

\section{B Saccade to target}

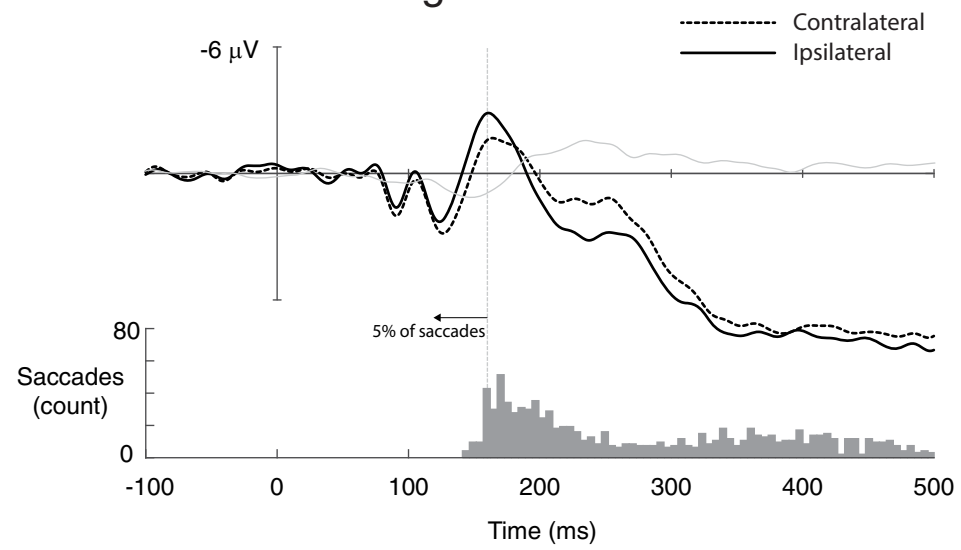

C Difference waves

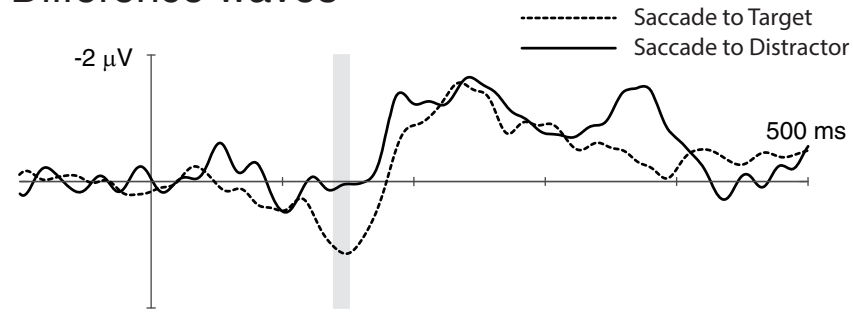

Figure 8. ERPs from Experiment 3 following a 'Ready' cue, when the target was on the vertical meridian and the distractor at a lateral location, time-locked to the onset of the search array. The top panel is a stylized representation of the search array; the stimulus with red color is denoted by capital letter. Histograms reflect the distribution of SRTs in each condition. $\boldsymbol{A}$, Posterior lateral ERPs elicited contralateral and ipsilateral to the distractor in trials where the eyes were deployed to the distractor. $\boldsymbol{B}$, Posterior lateral ERPs elicited contralateral and ipsilateral to the distractor in trials where the eyes were deployed to the target. $\mathbf{C}$, Contralatera-minus-ipsilateral difference waves.

Time-frequency analysis

Results from time-frequency analysis are illustrated in Figure 7. In the interval between the cue and search array, the cue created a broad increase in oscillatory power in the cortical hemisphere contralateral to the cued location.

As in experiment 1, we approached results from experiment 2 with the idea that cue-elicited oscillatory activity might predict selective processing of the distractor. To test this, we isolated cue trials and extracted trial-wise pretarget lateral alpha power (by subtracting ipsilateral power from contralateral power over the time-frequency interval identified by the broken box in Fig. 7A) and trial-wise post-target lateral EEG amplitude (over the time period identified by the gray rectangle in Fig. $6 C$; N2pc). We again rank transformed these values and used mixed linear modeling and model selection to assess their relationship. A simple initial model included a continuous fixed effect predictor for $\alpha$ power (oscillatory_power) and a random effect for the intercept of each participant (participant; AIC: 43526). This model was sequentially improved by adding a random effect for the per-participant $\alpha$ slope (AIC: 43499 ) and fixed factors for the additive effect of distractor elevation in the visual field and its interaction with $\alpha$ (distractor_elevation; AIC: 43498; Eq. 2). No model improvement resulted from the further inclusion of factors for the random effect of distractor elevation for each participant, the fixed effect of the target-distractor spatial proximity, the fixed effect of SRT, or the random slope of SRT for each participant.

$$
\begin{gathered}
\text { N2pc } \sim \text { oscillatory_power } * \\
\text { distractor_elevation } \\
+(1+\text { oscillatory_power } \mid \text { participant }) .
\end{gathered}
$$

ANOVA analysis identified a positive relationship between cue-elicited $\alpha$ and distractor-elicited lateral EEG $\left(F_{(1,27.8)}=7.86, p\right.$ $=0.009)$, with lateral $\alpha$ predicting a decrease in the negative-polarity distractor-elicited N2pc (Fig. 7B). Distractor elevation also had a significant effect on $\operatorname{EEG}\left(F_{(1,3733)}=2.88\right.$, $p=0.049$ ), with a distractor in the lower hemifield creating a larger N2pc. The effect of $\alpha$ interacted with distractor elevation $\left(F_{(1,3753.9)}=5.43, p=0.020\right)$, with cue-elicited $\alpha$ showing a stronger relationship with distractor-elicited N2pc when the distractor was in the lower hemifield. That the lateralized effect is more sensitive to prestimulus $\alpha$ when the eliciting stimulus is in the lower hemifield again suggests that variance in this brain activity reflects variance in N2pc as the N2pc emerges more robustly for lower-field visual stimuli, whereas the $P_{D}$ emerges more robustly for upper-field visual stimuli (Hickey et al., 2009; Tay et al., 2019).

\section{Experiment 3}

Experiments 1 and 2 demonstrated that the spatial distractor cue elicited strategic and proactive distractor suppression, which was indexed in cue-elicited lateral alpha power and reduced overt 
and covert selection of the distractor. Experiment 3 was designed to determine whether a feature cue could have a similar impact on distractor processing. In the critical distractor cue condition, participants were informed that the forthcoming distractor would be uniquely characterized by the red color (Fig. 1C). Our expectation was that the distractor cue would elicit proactive suppression of the red distractor.

\section{Behavior}

Trials that did not begin with fixation $(4.0 \%)$, that were anticipative responses $(0.7 \%)$, or that did not result in target or distractor selection (3.3\%) were removed from analysis.

Saccadic performance is illustrated in Figure 2E. Permutation contrasts demonstrated that in the ready cue condition, the apparent increase in saccadic selection of the target when the red color characterized the distractor rather than target was not statistically significant ( $p=0.095)$. However, accuracy did improve when the red distractor was preceded by a distractor cue rather than a ready cue $(p<0.001)$. SRT is illustrated in Figure $2 F$. A RANOVA with a factor for saccade direction (target vs distractor) and cue type (ready cue and red target; ready cue and red distractor; distractor cue) identified a main effect of saccade direction $\left(F_{(1,13)}=\right.$ 11.95, $p=0.004)$, reflecting faster onset of saccades to the distractor, and a main effect of cue $\left(F_{(2,13)}=17.15, p<0.001\right.$; interaction $F<1$ ), reflecting a combination of a general speeding when the distractor was red and a specific speeding in the distractor cue condition.

\section{ERPs}

As illustrated in Figure $8 A-C$, when participants made a saccade to a red target on the vertical meridian of the display after a ready cue, the ERP elicited over the contralateral visual cortex showed greater positive voltage in the interval preceding saccades compared with when participants deployed their eyes to the distractor. To test this difference we conducted a RANOVA with factors for electrode laterality (ipsilateral vs contralateral) and saccadic behavior (target directed vs distractor directed) based on mean ERP voltage in a $10 \mathrm{~ms}$ interval centered on the cross-conditional peak of the early lateral positivity (illustrated by gray box in Fig. 8C). This identified a critical interaction between electrode laterality and cue type $\left(F_{(1,13)}=7.47, p=0.017\right)$. A main effect of electrode laterality also emerged, reflecting the general reliability of the lateral positivity regardless of subsequent eye-movement behavior $\left(F_{(1,13)}=6.99, p=0.020\right.$; cue: $\left.F_{(1,13)}=2.50, p=0.138\right)$.

This finding reproduces results from Weaver et al. (2017; experiment 1 ), where this early positive component was also found ence waves.
Lateral red distractor / Saccade to target

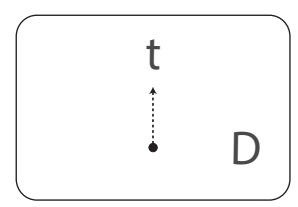

A 'Ready' cue

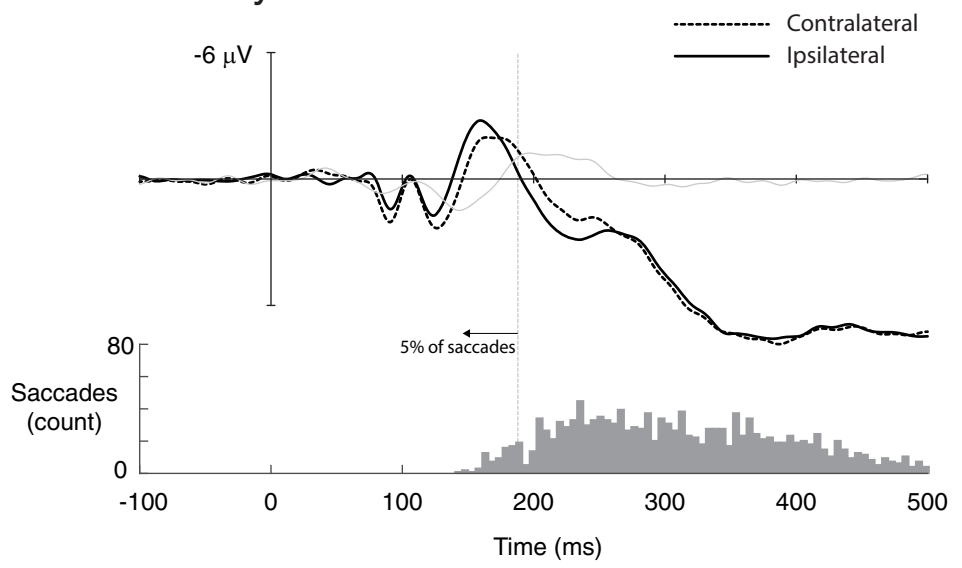

B 'Distractor' cue

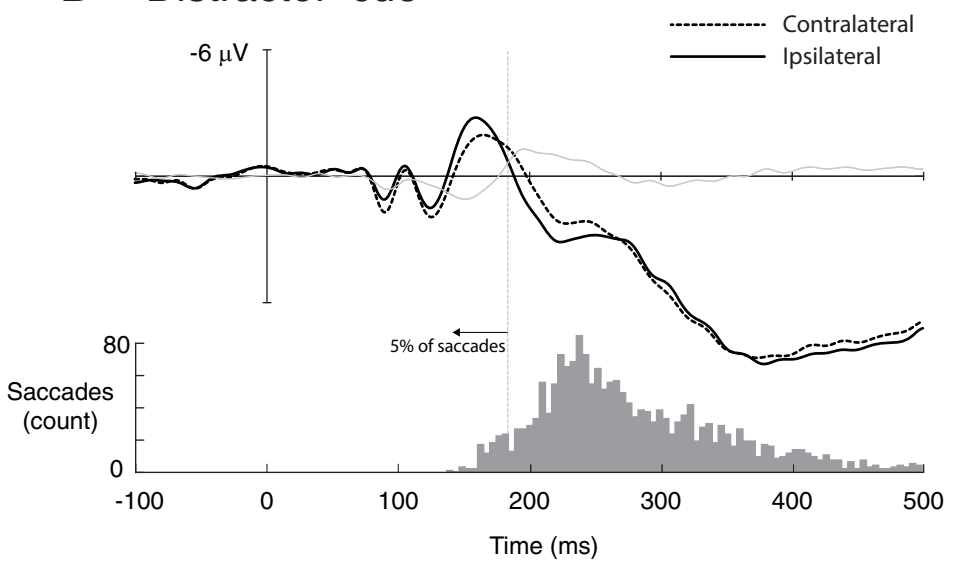

C Difference waves

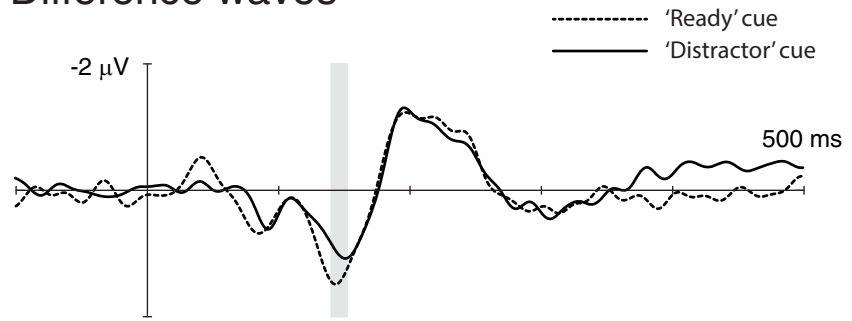

Figure 9. ERPs from Experiment 3 when the eyes were deployed to a target on the vertical meridian and a red distractor was successfully ignored. The top panel is a stylized representation of the search array; the stimulus with red color is denoted by capital letter. Histograms reflect the distribution of SRTs in each condition. $\boldsymbol{A}$, Posterior lateral ERPs elicited contralateral and ipsilateral to the distractor in trials following a 'Ready' cue. $\boldsymbol{B}$, Posterior lateral ERPs elicited contralateral and ipsilateral to the distractor in trials following a 'Distractor' cue. C, Contralateral-minus-ipsilateral differ-

to be larger when a lateral distractor was successfully ignored and the eyes were accurately deployed to a target on the vertical. This is important because early positive-polarity activity can emerge in the lateral ERP for a number of reasons, some of them possibly linked to sensory imbalances across the visual hemifields (Fortier- 


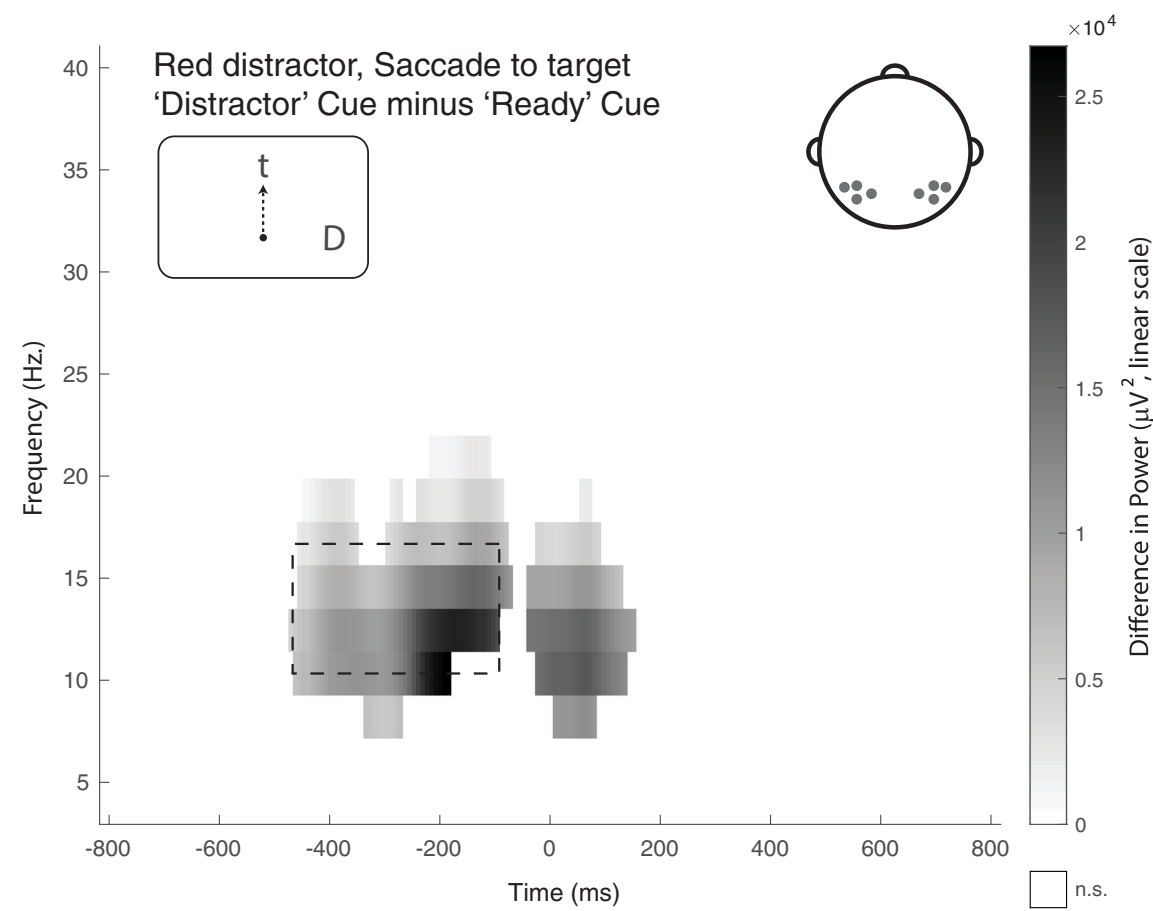

Figure 10. Posterior bilateral oscillatory power in Experiment 3. Shaded areas reflect time-frequency combinations where oscillatory power was greater in the 'Distractor' rather than 'Ready' cue condition. The time-frequency interval identified by broken box was employed in trial-wise analysis of the relationship between oscillatory power and EEG amplitude. Results that did not survive statistical correction are identified with n.s.

Gauthier et al., 2013; Jannati et al., 2013). The early $\mathrm{P}_{\mathrm{D}}$ observed here, and in Weaver et al. (2017), cannot be a product of sensory activity because, when elicited by the same stimulus display, it only emerges when the distractor is successfully ignored.

The results described above thus demonstrate the utility of the early $\mathrm{P}_{\mathrm{D}}$ as an index of distractor suppression. To determine the impact of the cue on this index of distractor suppression, we focused on results observed when participants made a correct saccade to a target on the vertical meridian, ignoring a lateral red distractor (Fig. 9A-C). Recent studies have found that when statistical learning (van Moorselaar and Slagter, 2019; van Moorselaar et al., 2020) or spatial cues (Heuer and Schubö, 2019) support distractor suppression before stimulus onset, the distractor-elicited $\mathrm{P}_{\mathrm{D}}$ reduces in amplitude. The idea here is that preemptive distractor suppression reduces the need for a suppressive response when the distractor actually appears. Results from experiment 3 support this notion, showing that the early $\mathrm{P}_{\mathrm{D}}$ is smaller in amplitude following a distractor cue rather than a ready cue.

To test the reduction in early $\mathrm{P}_{\mathrm{D}}$ amplitude we conducted a RANOVA with factors for electrode laterality and cue type (ready cue vs distractor cue) based on mean ERP voltage observed in a $10 \mathrm{~ms}$ interval centered on the cross-conditional peak of the early $\mathrm{P}_{\mathrm{D}}$ (gray box in Fig. 9C). This identified a critical interaction between electrode laterality and cue type $\left(F_{(1,13)}=\right.$ $6.28, p=0.026)$, reflecting the reliable decrease in early $\mathrm{P}_{\mathrm{D}}$ amplitude in the distractor cue condition. An additional main effect of electrode laterality was identified $\left(F_{(1,13)}=8.17, p=0.013\right)$, demonstrating the general reliability of the early $\mathrm{P}_{\mathrm{D}}$ across cuing conditions (cue: $\left.F_{(1,13)}=4.03, p=0.066\right)$.

\section{Time-frequency analysis}

As illustrated in Figure 10, when trials were preceded by a distractor cue rather than a ready cue, and participants therefore knew with certainty that the red color would characterize the distractor stimulus, bilateral oscillatory activity in the pretarget interval increased through the $\alpha$ (8$12.5 \mathrm{~Hz}$ ) and low $\beta$ range $(12.5-25 \mathrm{~Hz})$.

As in experiment 2, we approached results with the idea that this oscillatory activity might predict variance in distractor processing as indexed in the post-target ERP. Our specific expectation was that prestimulus suppression, as indexed in oscillatory power, might reduce the need for poststimulus distractor suppression, as indexed in early $\mathrm{P}_{\mathrm{D}}$. We tested for this by (1) extracting power across a frequency band $(10.4-16.7 \mathrm{~Hz})$ and latency interval ( -480 to $-60 \mathrm{~ms}$ ) where cue type had a significant impact on oscillatory power, and (2) extracting mean EEG amplitude in a $10 \mathrm{~ms}$ posttarget latency period centered on the cross-conditional peak of the early $\mathrm{P}_{\mathrm{D}}$ (gray box in Fig. 9C).

As in earlier experiments, we rank transformed these values and used mixed linear modeling and model selection to identify the relationship between cue-elicited oscillatory power and the early $\mathrm{P}_{\mathrm{D}}$. An initial model contained a continuous fixed effect predictor for oscillatory power and a random effect for the intercept of each participant (AIC: 18217). This model was sequentially improved by adding a fixed effect for SRT and the interaction of oscillatory power with SRT (AIC: 18201), and random effects for the per-participant $\alpha$ intercept and slope (AIC:18199; Eq. 3). Inclusion of per-participant intercept and slope for SRT did not lead to model improvement.

$$
\begin{gathered}
\text { early_ } \mathrm{P}_{\mathrm{D}} \sim \text { oscillatory_power } \\
* \mathrm{SRT}+(1+\text { oscillatory_power } \mid \text { participant }) .
\end{gathered}
$$

ANOVA analysis of this model identified a positive relationship between cue-elicited oscillatory power and EEG amplitude $\left(F_{(1,500.3)}=7.21, p=0.007\right)$, with cue-elicited $\alpha / \beta$ predicting an increase in amplitude of the early $\mathrm{P}_{\mathrm{D}}$. SRT also had a significant positive effect on EEG $\left(F_{(1,1651.6)}=22.71, p<\right.$ $0.001)$, with larger amplitude early $P_{D}$ associated to slower saccade onset. Critically, these effects interacted $\left(F_{(1,1705.2)}=13.30, p<\right.$ $0.001)$.

To gain further insight on this interaction we conducted a median split based on SRT and fit each of the resulting datasets with a simple model containing a fixed effect for oscillatory power and a random effect for the per-participant intercept as follows:

$$
\begin{gathered}
\text { early_P } \mathrm{P}_{\mathrm{D}} \sim \text { oscillatory_power } \\
+(1 \mid \text { participant }) .
\end{gathered}
$$

In analysis of short-latency SRT data, the relationship between oscillatory power and early $\mathrm{P}_{\mathrm{D}}$ amplitude was negative so that oscillatory power predicted a smaller $\mathrm{P}_{\mathrm{D}}$ (parameter 
estimate: -0.0277), whereas in an analysis of long-latency SRT data, the relationship between oscillatory power and early $\mathrm{P}_{\mathrm{D}}$ was positive in that oscillatory power predicted a larger $\mathrm{P}_{\mathrm{D}}$ (parameter estimate: 0.0245 ). Bilateral $\alpha$ thus predicted a reduction in early $\mathrm{P}_{\mathrm{D}}$ when participants responded quickly but an increase in early $\mathrm{P}_{\mathrm{D}}$ when participants responded at longer latency.

\section{Discussion}

We demonstrate that foreknowledge of distractor characteristics leads to strategic, proactive distractor suppression. Experiments 1 and 2 used a spatial cue, showing that the eyes were less likely to be deployed to a cued distractor when the target and distractor appeared in close spatial proximity. In both experiments, this change in overt behavior was preceded by a reduction of the distractor-elicited N2pc, reflecting a decrease in attentional selection of the distractor. In experiment 1 , analysis of saccade deviation suggested that cue-elicited distractor suppression was implemented before onset of the search array. In experiment 2, this proactive suppression was linked to the emergence of cueelicited lateral $\alpha$. As the target only ever appeared on the vertical meridian of the display, but the $\alpha$ effect was lateralized, the effect cannot be easily linked to monitoring of target location or other target-related processes. Analysis showed that the trial-wise magnitude of pretarget lateral $\alpha$ predicted trial-wise reduction of post-target distractor-elicited N2pc. Participants therefore appear able to strategically and proactively suppress distractors at a cued location, eliciting lateral $\alpha$, and this reduces the propensity to covertly and overtly select the distractor when it appears.

Experiment 3 investigated the impact of a cue identifying a unique distractor feature. The distractor cue identified with $100 \%$ validity that the distractor would be red, whereas the ready cue indicated there was a $50 \%$ chance the distractor would be red and a $50 \%$ chance the target would be red. The distractor cue led to a decrease in the proportion of saccades to the distractor. When the eyes were deployed to the target, post-target ERPs showed emergence of an early distractor-elicited $\mathrm{P}_{\mathrm{D}}$, reflecting online, stimulus-triggered suppression of the lateral distractor. The cue reduced the size of this early $\mathrm{P}_{\mathrm{D}}$, suggesting that cue-elicited suppression in the pretarget interval limited the need for stimulus-triggered suppression when the distractor appeared.

The semantic cue used in experiment 3 created an increase in bilateral $\alpha /$ low beta power, conceptually reproducing earlier results (Payne et al., 2013), and trial-wise variance in this signal predicted post-target distractor-elicited early $\mathrm{P}_{\mathrm{D}}$. Bilateral $\alpha$ over the posterior cortex has been interpreted as a general downweighting of perceptual input (Bonnefond and Jensen, 2012; de Vries et al., 2019), and this is consistent with results showing that it predicts a decrease in the need for stimulus-triggered distractor suppression, as indexed in early $\mathrm{P}_{\mathrm{D}}$, when participants make quick saccadic response to the target. However, the cue also improves target-directed saccadic accuracy and speed, which is hard to reconcile with the idea that all stimuli representations have been degraded. One alternative is that bilateral $\alpha$ contributes to perceptual down-weighting of the cued distractor specifically, leaving the target unaffected (Folk and Remington, 1998). This suppression could act to reduce the salience of a discrete feature-redness in our experiment-or could suppress the entire feature dimension so that discontinuities along this dimension do not pop out (Liesefeld and Müller, 2019).

Why do we see unambiguous evidence of strategic, proactive distractor suppression, where prominent recent studies have not? A critical determinant appears to be that the target and distractor are defined within the same featural dimension. That is, the target and distractor in our experiments were rendered salient by orientation, and direction or magnitude of orientation played a role in defining which stimulus was the target. Studies showing an effect of proactive distractor suppression tend to define target and distractor within the same dimension (Ruff and Driver, 2006; Munneke et al., 2008, 2011). In contrast, Wang and Theeuwes (2018) failed to find an effect of a distractor cue when the target was consistently defined by unique form and the salient distractor by unique color. Visual processing is segregated so that retinotopic brain areas respond preferentially to discrete stimuli features like color, orientation, and motion (Zeki and Shipp, 1988), and the definition of targets and distractors within the same featural dimension increases distractor interference (Liesefeld and Müller, 2019). One possibility is therefore that proactive distractor suppression is used only when target-distractor similarity is high (van Zoest and Donk, 2008; Conci et al., 2019) and distractor representations intrude on target representations within the same dimension-specific cortical areas.

Competition created by physical proximity also appears to play an important role in determining how proactive distractor suppression has an impact on behavior and brain activity. Experiments 1 and 2 show that proactive distractor suppression of a cued location affects covert and overt selection of the distractor only when the target and distractor appear in close spatial proximity. This is consistent with what we know of distractor suppression more broadly. Although the existence of strategic, proactive distractor suppression has been debated, distractor suppression during target selection is widely accepted as a core mechanism in the resolution of target information (Moran and Desimone, 1985; Chelazzi et al., 1993; Luck et al., 1997a). This 'secondary' distractor suppression (Noonan et al., 2018) occurs during target resolution and increases as the distance between target and distractor reduces (Mounts, 2000; Hopf et al., 2006; Hilimire et al., 2011; Hickey and Theeuwes, 2011), reflecting an increasing need for the resolution of neural ambiguity as the stimuli come to stimulate an overlapping set of retinotopic neurons (Desimone and Duncan, 1995; Tsotsos et al., 1995; Luck et al., 1997b). The relationship between spatial competition and distractor suppression identified in our data is important to the interpretation of extant results. A prominent failure to find an effect of a spatial distractor cue used a design with targets and distractors presented either to separate upper and lower visual hemifields or separate left and right hemifields (Noonan et al., 2016). That design was therefore similar to conditions of experiments 1 and 2, where the target and distractor appeared distant from one another, and where we found no impact of the distractor cue on behavior or post-target brain activity. Importantly, we find here that a spatial distractor cue does have an impact on distractor processing when the target and distractor appear in closer proximity.

A final possibility is that strategic, proactive distractor suppression might develop from a basis of implicit learning. In extant studies, targets and distractors tend to appear with equal frequency at the same locations. In contrast, in experiments 1 and 2, distractors appeared at lateral locations where targets never appeared, and participants were never required to select stimuli at these locations. Over the course of $\sim 800$ trials, participants had the opportunity to learn how to ignore stimuli at these locations. Similarly, in experiment 3, the red color more commonly characterized the distractor than it did the target, giving participants the opportunity to learn how to effectively suppress 
red objects. This prior experience may be required for volitional control of suppression to emerge. That is, suppression of specific features or locations may become strategically accessible only once this cognitive operation is familiar, unambiguous, and well practiced, and when characteristics of distractors do not commonly overlap with characteristics of targets.

Cue-elicited $\alpha$ emerged in all three experiments and, on the face of it, this conflicts with a developing literature looking at the impact of prediction and statistical learning on distractor suppression (Ferrante et al., 2018; Wang and Theeuwes, 2018; Won and Geng, 2020). Distractor suppression fostered by statistical learning does not appear to be associated with $\alpha$ (Noonan et al., 2016; van Moorselaar and Slagter, 2019, 2020; but see Wang et al. 2019) and this has contributed to a wholescale discounting of the relationship between $\alpha$ and distractor suppression (Noonan et al., 2018; Foster and Awh, 2019; van Moorselaar and Slagter, 2020). This may be premature. It seems possible that the distractor suppression created by statistical learning may be qualitatively different from that created by strategy. Statistical learning of distractor status over repeated experience could rely on slow mechanisms, like synaptic plasticity, and involve long-range neuromodulatory architecture (Roelfsema and van Ooyen, 2005). This kind of latent distractor suppression, instantiated in synaptic weighting between idle cells, would not necessarily be associated with a brain signal in the cue-target interval. In contrast, strategic, cue-elicited distractor suppression must be implemented in the cue-target interval and is therefore more likely to elicit discernible brain activity in this time.

A particular challenge to the relationship between $\alpha$ and distractor suppression has come from studies using frequency-tagged visual stimuli. In frequency-tagging experiments, stimuli are presented with oscillating contrast at a specific frequency, generating a neural response with corresponding frequency. When stimuli are attended, this brain signal increases in power (Müller et al., 1998a; but see Adam et al., 2020), which leads to the reasonable expectation that when stimuli are suppressed, generating lateral $\alpha$, the tagged brain signal should decrease in power. But this is not what is observed (Antonov et al., 2020; Zhigalov and Jensen, 2020). Results from the current study provide some insight on this null relationship. We find that distractor suppression predicts variance in lateralized brain responses associated with relatively high-level visual cortex (Hopf et al., 2000). In contrast, the oscillatory signal induced by frequency tagging tends to emerge over the occipital pole (Müller et al., 1998b), suggesting that it originates from early visual cortex (Di Russo et al., 2007). This raises the possibility that distractor suppression indexed in lateral $\alpha$ has an impact on stimuli representations in visual areas that simply do not express the frequency-tagging signal (Zhigalov and Jensen, 2020).

In summary, we present unambiguous evidence that strategic, proactive suppression of visual distractors leads to attenuated attentive and oculomotor responses to these stimuli and that this suppression is linked to the power of prestimulus $\alpha$. Knowing the characteristics of visual distractors helps you ignore them.

\section{References}

Adam KC, Chang L, Rangan N, Serences JT (2020) Steady-state visually evoked potentials and feature-based attention: preregistered null results and a focused review of methodological considerations. J Cognitive Neurosci 33:695-724.
Antonov PA, Chakravarthi R, Andersen SK (2020) Too little, too late, and in the wrong place: Alpha band activity does not reflect an active mechanism of selective attention. Neuroimage 219:117006.

Arita JT, Carlisle NB, Woodman GF (2012) Templates for rejection: configuring attention to ignore task-irrelevant features. J Exp Psychol Hum Percept Perform 38:580-584.

Awh E, Matsukura M, Serences JT (2003) Top-down control over biased competition during covert spatial orienting. J Exp Psychol Hum Percept Perform 29:52-63.

Beck VM, Luck SJ, Hollingworth A (2018) Whatever you do, don't look at the...: Evaluating guidance by an exclusionary attentional template. J Exp Psychol Hum Percept Perform 44:645-662.

Becker MW, Hemsteger S, Peltier C (2015) No templates for rejection: A failure to configure attention to ignore task-irrelevant features. Visual Cognition 23:1150-1167.

Bell AJ, Sejnowski TJ (1995) An information-maximization approach to blind separation and blind deconvolution. Neural Comput 7:1129-1159.

Bonnefond M, Jensen O (2012) Alpha oscillations serve to protect working memory maintenance against anticipated distracters. Curr Biol 22:19691974.

Brainard DH (1997) The psychophysics toolbox. Spat Vis 10:433-436.

Carlisle NB, Woodman GF (2011) Automatic and strategic effects in the guidance of attention by working memory representations. Acta Psychol (Amst) 137:217-225.

Chang S, Cunningham CA, Egeth HE (2019) The power of negative thinking: Paradoxical but effective ignoring of salient-but-irrelevant stimuli with a spatial cue. Visual Cognition 27:199-213.

Chao HF (2010) Top-down attentional control for distractor locations: he benefit of precuing distractor locations on target localization and discrimination. J Exp Psychol Hum Percept Perform 36:303-316.

Chelazzi L, Miller EK, Duncan J, Desimone R (1993) A neural basis for visual search in inferior temporal cortex. Nature 363:345-347.

Chelazzi L, Marini F, Pascucci D, Turatto M (2019) Getting rid of visual distractors: the why, when, how, and where. Curr Opin Psychol 29:135-147.

Conci M, Deichsel C, Müller H, Töllner T (2019) Feature guidance by negative attentional templates depends on search difficulty. Visual Cognition 27:317-326.

Conover WJ, Iman RL (1981) Rank transformations as a bridge between parametric and nonparametric statistics. Am Stat 35:124129.

Cousineau D (2005) Confidence intervals in within-subject designs: A simpler solution to Loftus and Masson's method. Tutor Quant Methods Psychol 1:42-45.

Cunningham CA, Egeth HE (2016) Taming the white bear: Initial costs and eventual benefits of distractor inhibition. Psychol Sci 27:476485.

de Vries IE, Savran E, van Driel J, Olivers CN (2019) Oscillatory mechanisms of preparing for visual distraction. J Cogn Neurosci 31:1873-1894.

Delorme A, Makeig S (2004) EEGLAB: an open source toolbox for analysis of single-trial EEG dynamics including independent component analysis. J Neurosci Methods 134:9-21.

Desimone R, Duncan J (1995) Neural mechanisms of selective visual attention. Annu Rev Neurosci 18:193-222.

Di Russo F, Pitzalis S, Aprile T, Spitoni G, Patria F, Stella A, Spinelli D, Hillyard SA (2007) Spatiotemporal analysis of the cortical sources of the steady-state visual evoked potential. Hum Brain Mapp 28:323-334.

Dimigen O, Sommer W, Hohlfeld A, Jacobs AM, Kliegl R (2011) Coregistration of eye movements and EEG in natural reading: analyses and review. J Exp Psychol Gen 140:552-572.

Dombrowe I, Hilgetag CC (2014) Occipitoparietal alpha-band responses to the graded allocation of top-down spatial attention. J Neurophysiol 112:1307-1316.

Ferrante O, Patacca A, Di Caro V, Della Libera C, Santandrea E, Chelazzi L (2018) Altering spatial priority maps via statistical learning of target selection and distractor filtering. Cortex 102:67-95.

Folk CL, Remington R (1998) Selectivity in distraction by irrelevant featural singletons: evidence for two forms of attentional capture. J Exp Psychol Hum Percept Perform 24:847.

Fortier-Gauthier U, Dell'Acqua R, Jolicóur P (2013) The "red-alert" effect in visual search: Evidence from human electrophysiology. Psychophysiol 50:671-679. 
Foster JJ, Awh E (2019) The role of alpha oscillations in spatial attention: limited evidence for a suppression account. Curr Opin Psychol 29:34-40.

Foxe JJ, Snyder AC (2011) The role of alpha-band brain oscillations as a sensory suppression mechanism during selective attention. Front Psychol 2:154.

Gaspelin N, Luck SJ (2019) Inhibition as a potential resolution to the attentional capture debate. Curr Opin Psychol 29:12-18.

Haegens S, Nácher V, Luna R, Romo R, Jensen O (2011) $\alpha$-Oscillations in the monkey sensorimotor network influence discrimination performance by rhythmical inhibition of neuronal spiking. Proc Natl Acad Sci U S A 108:19377-19382.

Händel BF, Haarmeier T, Jensen O (2011) Alpha oscillations correlate with the successful inhibition of unattended stimuli. J Cogn Neurosci 23:2494-2502.

Heuer A, Schubö A (2019) Cuing distraction: electrophysiological evidence for anticipatory active suppression of distractor location. Psychol Res $84: 2111-2121$

Hickey C, Theeuwes J (2011) Context and competition in the capture of visual attention. Atten Percept Psychophys 73:2053-2064.

Hickey C, Van Zoest W (2012) Reward creates oculomotor salience. Curr Biol 22:R219-R220.

Hickey C, McDonald JJ, Theeuwes J (2006) Electrophysiological evidence of the capture of visual attention. J Cogn Neurosci 18:604-613.

Hickey C, Di Lollo V, McDonald JJ (2009) Electrophysiological indices of target and distractor processing in visual search. J Cogn Neurosci 21:760775 .

Hilimire MR, Mounts JR, Parks NA, Corballis PM (2011) Dynamics of target and distractor processing in visual search: Evidence from event-related brain potentials. Neurosci Lett 495:196-200.

Hilimire MR, Hickey C, Corballis PM (2012) Target resolution in visual search involves the direct suppression of distractors: evidence from electrophysiology. Psychophysiology 49:504-509.

Hopf JM, Luck SJ, Girelli M, Hagner T, Mangun GR, Scheich H, Heinze HJ (2000) Neural sources of focused attention in visual search. Cereb Cortex 10:1233-1241.

Hopf JM, Boehler CN, Luck SJ, Tsotsos JK, Heinze HJ, Schoenfeld MA (2006) Direct neurophysiological evidence for spatial suppression surrounding the focus of attention in vision. Proc Natl Acad Sci USA 103:1053-1058.

Iman RL, Conover WJ (1979) The use of the rank transform in regression. Technometrics 21:499-509.

Jannati A, Gaspar JM, McDonald JJ (2013) Tracking target and distractor processing in fixed-feature visual search: evidence from human electrophysiology. J Exp Psychol Hum Percept Perform 39:1713-1730.

Jensen O, Mazaheri A (2010) Shaping functional architecture by oscillatory alpha activity: gating by inhibition. Front Hum Neurosci 4:186.

Klimesch W, Sauseng P, Hanslmayr S (2007) EEG alpha oscillations: the inhibition-timing hypothesis. Brain Res Rev 53:63-88.

Liesefeld HR, Müller HJ (2019) Distractor handling via dimension weighting. Curr Opin Psychol 29:160-167.

Luck SJ, Hillyard SA (1994a) Electrophysiological correlates of feature analysis during visual search. Psychophysiology 31:291-308

Luck SJ, Hillyard SA (1994b) Spatial filtering during visual search: evidence from human electrophysiology. J Exp Psychol Hum Percept Perform 20:1000-1014

Luck SJ, Chelazzi L, Hillyard SA, Desimone R (1997a) Neural mechanisms of spatial selective attention in areas V1, V2, and V4 of macaque visual cortex. J Neurophysiol 77:24-42.

Luck SJ, Girelli M, McDermott MT, Ford MA (1997b) Bridging the gap between monkey neurophysiology and human perception: An ambiguity resolution theory of visual selective attention. Cogn Psychol 33:64-87.

Luck SJ, Gaspelin N, Folk CL, Remington RW, Theeuwes J (2021) Progress toward resolving the attentional capture debate. Vis cogn 29:1-21.

Mathôt S, Hickey C, Theeuwes J (2010) From reorienting of attention to biased competition: Evidence from hemifield effects. Atten Percept Psychophys 72:651-657.

McPeek RM, Han JH, Keller EL (2003) Competition between saccade goals in the superior colliculus produces saccade curvature. J Neurophysiol $89: 2577-2590$
Moher J, Egeth HE (2012) The ignoring paradox: Cuing distractor features leads first to selection, then to inhibition of to-be-ignored items. Atten Percept Psychophys 74:1590-1605

Moran J, Desimone R (1985) Selective attention gates visual processing in the extrastriate cortex. Science 229:782-784.

Mounts JR (2000) Attentional capture by abrupt onsets and feature singletons produces inhibitory surrounds. Percept Psychophys 62:1485-1493.

Mulckhuyse M, Van der Stigchel S, Theeuwes J (2009) Early and late modulation of saccade deviations by target distractor similarity. J Neurophysiol 102:1451-1458.

Müller MM, Teder-Sälejärvi W, Hillyard SA (1998a) The time course of cortical facilitation during cued shifts of spatial attention. Nat Neurosci 1:631-634.

Müller MM, Picton TW, Valdes-Sosa P, Riera J, Teder-Sälejärvi WA, Hillyard SA (1998b) Effects of spatial selective attention on the steadystate visual evoked potential in the $20-28 \mathrm{~Hz}$ range. Cogn Brain Res 6:249-261.

Munneke J, Van der Stigchel S, Theeuwes J (2008) Cuing the location of a distractor: An inhibitory mechanism of spatial attention? Acta Psychol (Amst) 129:101-107.

Munneke J, Heslenfeld DJ, Usrey WM, Theeuwes J, Mangun GR (2011) Preparatory effects of distractor suppression: Evidence from visual cortex. PloS one 6:e27700.

Noonan MP, Adamian N, Pike A, Printzlau F, Crittenden BM, Stokes MG (2016) Distinct mechanisms for distractor suppression and target facilitation. J Neurosci 36:1797-1807.

Noonan MP, Crittenden BM, Jensen O, Stokes MG (2018) Selective inhibition of distracting input. Behav Brain Res 355:36-47.

Oostenveld R, Fries P, Maris E, Schoffelen JM (2011) FieldTrip: open source software for advanced analysis of MEG, EEG, and invasive electrophysiological data. Comput Intell Neurosci 2011:156869.

Payne L, Guillory S, Sekuler R (2013) Attention-modulated alpha-band oscillations protect against intrusion of irrelevant information. J Cogn Neurosci 25:1463-1476.

Plöchl M, Ossandón JP, König P (2012) Combining EEG and eye tracking: identification, characterization, and correction of eye movement artifacts in electroencephalographic data. Front Hum Neurosci 6:278.

Reeder RR, Olivers CN, Pollmann S (2017) Cortical evidence for negative search templates. Vis Cogn 25:278-290.

Roelfsema PR, van Ooyen A (2005) Attention-gated reinforcement learning of internal representations for classification. Neural Comput 17:21762214.

Ruff CC, Driver J (2006) Attentional preparation for a lateralized visual distractor: Behavioral and fMRI evidence. J Cogn Neurosci 18:522-538.

Sauseng P, Klimesch W, Stadler W, Schabus M, Doppelmayr M, Hanslmayr S, Gruber WR, Birbaumer N (2005) A shift of visual spatial attention is selectively associated with human EEG alpha activity. Eur J Neurosci 22:2917-2926.

Sawaki R, Luck SJ (2010) Capture versus suppression of attention by salient singletons: Electrophysiological evidence for an automatic attend-to-me signal. Atten Percept Psychophys 72:1455-1470.

Scheeringa R, Petersson KM, Kleinschmidt A, Jensen O, Bastiaansen MC (2012) EEG alpha power modulation of fMRI resting-state connectivity. Brain Connect 2:254-264.

Spaak E, Bonnefond M, Maier A, Leopold DA, Jensen O (2012) Layer-specific entrainment of gamma-band neural activity by the alpha rhythm in monkey visual cortex. Curr Biol 22:2313-2318.

Tay D, Harms V, Hillyard SA, McDonald JJ (2019) Electrophysiological correlates of visual singleton detection. Psychophysiology 56: e13375.

Thut G, Nietzel A, Brandt SA, Pascual-Leone A (2006) $\alpha$-Band electroencephalographic activity over occipital cortex indexes visuospatial attention bias and predicts visual target detection. J Neurosci 26:9494-9502.

Tsotsos JK, Culhane SM, Wai WYK, Lai Y, Davis N, Nuflo F (1995) Modeling visual attention via selective tuning. Artificial intelligence 78:507-545

Van der Stigchel S, Theeuwes J (2006) Our eyes deviate away from a location where a distractor is expected to appear. Exp Brain Res 169:338-349.

Van der Stigchel S, Meeter M, Theeuwes J (2006) Eye movement trajectories and what they tell us. Neurosci Biobehav Rev 30:666-679. 
van Moorselaar D, Slagter HA (2019) Learning what is irrelevant or relevant: Expectations facilitate distractor inhibition and target facilitation through distinct neural mechanisms. J Neurosci 39:6953-6967.

van Moorselaar D, Lampers E, Cordesius E, Slagter HA (2020) Neural mechanisms underlying expectation-dependent inhibition of distracting information. Elife 9:e61048

van Moorselaar D, Slagter HA (2020) Inhibition in selective attention. Ann N Y Acad Sci 1464:204-221.

van Zoest W, Donk M (2008) Goal-driven modulation as a function of time in saccadic target selection. Q J Exp Psychol 61:1553-1572.

van Zoest W, Donk M, Theeuwes J (2004) The role of stimulus-driven and goal-driven control in saccadic visual selection. J Exp Psychol Hum Percept Perform 30:746-759.

Wang B, Theeuwes J (2018) How to inhibit a distractor location? Statistical learning versus active, top-down suppression. Atten Percept Psychophys 80:860-870.

Wang B, van Driel J, Ort E, Theeuwes J (2019) Anticipatory distractor suppression elicited by statistical regularities in visual search. J Cogn Neurosci 31:1535-1548.
Weaver MD, van Zoest W, Hickey C (2017) A temporal dependency account of attentional inhibition in oculomotor control. Neuroimage 147:880894

Won B-Y, Geng JJ (2020) Passive exposure attenuates distraction during visual search. J Exp Psychol Gen 149:1987-1995.

Woodman GF, Luck SJ (2003) Serial deployment of attention during visual search. J Exp Psychol Hum Percept Perform 29:121-138.

Woodman GF, Luck SJ (2007) Do the contents of visual working memory automatically influence attentional selection during visual search? J Exp Psychol Hum Percept Perform 33:363-377.

Worden MS, Foxe JJ, Wang N, Simpson GV (2000) Anticipatory biasing of visuospatial attention indexed by retinotopically specific $\alpha$-bank electroencephalography increases over occipital cortex. J Neurosci 20:RC63-RC63.

Zeki S, Shipp S (1988) The functional logic of cortical connections. Nature 335:311-317.

Zhigalov A, Jensen O (2020) Alpha oscillations do not implement gain control in early visual cortex but rather gating in parieto-occipital regions. Hum Brain Mapp 41:5176-5186. 